ARTICLE

Received 4 Dec 2015 | Accepted 7 Feb 2016 | Published 21 Mar $2016 \quad$ DOl: 10.1038/ncomms10966 OPEN

\title{
Cajal bodies are linked to genome conformation
}

\author{
Qiuyan Wang ${ }^{1,2, \dagger}, \star$, lain A. Sawyer ${ }^{1,2, \star}$, Myong-Hee Sung ${ }^{2, \star}$, David Sturgill ${ }^{2, \star}$, Sergey P. Shevtsov ${ }^{1}$ \\ Gianluca Pegoraro 2,3 , Ofir Hakim ${ }^{2, \dagger}$, Songjoon Baek ${ }^{2}$, Gordon L. Hager ${ }^{2} \&$ Miroslav Dundr ${ }^{1}$
}

The mechanisms underlying nuclear body (NB) formation and their contribution to genome function are unknown. Here we examined the non-random positioning of Cajal bodies (CBs), major NBs involved in spliceosomal snRNP assembly and their role in genome organization. CBs are predominantly located at the periphery of chromosome territories at a multi-chromosome interface. Genome-wide chromosome conformation capture analysis (4C-seq) using CB-interacting loci revealed that CB-associated regions are enriched with highly expressed histone genes and $U$ small nuclear or nucleolar RNA (sn/snoRNA) loci that form intra- and inter-chromosomal clusters. In particular, we observed a number of CB-dependent gene-positioning events on chromosome 1. RNAi-mediated disassembly of $\mathrm{CBs}$ disrupts the CB-targeting gene clusters and suppresses the expression of $U$ sn/snoRNA and histone genes. This loss of spliceosomal snRNP production results in increased splicing noise, even in CB-distal regions. Therefore, we conclude that CBs contribute to genome organization with global effects on gene expression and RNA splicing fidelity.

\footnotetext{
${ }^{1}$ Department of Cell Biology, Rosalind Franklin University of Medicine and Science, Chicago Medical School, North Chicago, 60064 Ilinois, USA. ${ }^{2}$ Laboratory of Receptor Biology and Gene Expression, Center for Cancer Research, National Cancer Institute, National Institutes of Health, Bethesda, 20892 Maryland, USA. ${ }^{3}$ High-Throughput Imaging Facility (HiTIF), Center for Cancer Research, National Cancer Institute, National Institutes of Health, Bethesda, 20892 Maryland, USA. * These authors contributed equally to this work. † Present addresses: Center for Genomic and Personalized Medicine, Guangxi Medical University, Nanning, Guangxi 530021, China (Q.W.); The Mina and Everard Goodman Faculty of Life Sciences, Bar-Ilan University, Ramat-Gan 5290002, Israel (O.H.). Correspondence and requests for materials should be addressed to G.L.H. (email: hagerg@dce41.nci.nih.gov) or to M.D. (email: mirek.dundr@rosalindfranklin.edu).
} 
O ver the last decade it has become apparent that gene expression is regulated by the dynamic interplay between spatial genome organization and nuclear architecture. Within the membrane-free nuclear interior, chromosomes occupy spatially confined territories that intermingle near their edges $^{1,2}$. These territories adopt discrete non-random positions with some chromosomes preferentially situated in the interior and others near the periphery. Moreover, chromatin domains with a similar status of activity are found to cluster into higher order compartments ${ }^{3}$. This can lead to the formation of topological superdomains composed of multiple functionally related genomic loci ${ }^{4}$. In addition, a variety of nuclear bodies (NBs) exist, including the nucleoli, Cajal bodies (CBs), histone locus bodies (HLBs), PML NBs, splicing speckles, paraspeckles and nuclear stress bodies ${ }^{5-9}$. Several major NBs have been observed to nucleate at sites of active transcription with highly abundant transcripts or specific genomic regulatory elements functioning as a seeding scaffold in their formation ${ }^{10-13}$. Hence, these structures may arise by dynamic self-organization after an initial nucleation event, reflecting site-specific activities associated with gene expression and genome maintenance.

It has been suggested that molecular-crowding effects within NBs such as CBs are beneficial for efficient gene expression and NB structural maintenance ${ }^{14,15}$. They restrict the reaction volume to promote macromolecular complex assembly and association with substrates ${ }^{16}$. CBs have been implicated in the processing, assembly, modification and final maturation of spliceosomal small nuclear ribonucleic particles (snRNPs) and small nucleolar ribonucleic particles, biogenesis of telomerase, $3^{\prime}$-end processing of histone pre-mRNAs ${ }^{17-19}$, as well as in recycling of spliceosome components after each round of pre-mRNA splicing ${ }^{20,21}$.

Previous studies have documented physical associations of CBs with a limited number of major and minor spliceosomal snRNA genes and U3 snoRNA (SNORD3A) genes ${ }^{22-26}$. The relevance of this finding to snoRNA gene function is uncertain. More than $90 \%$ of human $U$ snoRNAs are encoded within the introns of protein-coding and non-coding host genes ${ }^{27}$, whereas the genes encoding the major U3, U8 and U14 snoRNAs (involved in pre-rRNA processing) have autonomous promoters located in intergenic regions. Similarly, some replication-dependent histone genes have been found to be associated with CBs, likely via the physically associated $\mathrm{HLB}^{28-30}$. However, complete characterization of the association of $\mathrm{CBs}$ with the genome and delineation of the functionally relevant interactions are lacking from existing data.

To understand the nature and extent of CB-proximal genome-genome interactions, we used 4C-seq analysis to characterize genome-wide contacts of $\mathrm{CB}$-associated genes in human cells. Combining $4 \mathrm{C}$ analysis with a novel six-colour co-localization DNA FISH imaging and high-throughput automated microscopy screening, we define a genome-wide chromatin interface with CBs. In particular, we identify a complex CB-proximal genome-interaction hub on chromosome 1. Our integrative analysis reveals $\mathrm{CB}$-dependent looping of all categories of sn/snoRNA, small CB-specific RNA (scaRNA) and histone gene loci across the genome into transcriptionally highly active intra- and inter-chromosomal regions. These interactions are greatly reduced in cells where CBs are absent or have been disassembled by specific depletions of essential CB components by RNAi. Small-and total-RNA-seq analyses show critical roles of CBs for the global maintenance of elevated sn/snoRNA expression in CB-containing aneuploidy cells, RNA pol II-driven transcription and splicing that are not limited to $\mathrm{CB}$-proximal genomic regions. Our study demonstrates that $\mathrm{CBs}$ contribute to topological chromosome organization and affect the gene expression of target RNAs through proximal association with specific sn/snoRNA gene loci. This ultimately influences RNA maturation processes and splicing fidelity.

\section{Results}

CB position relative to chromosomes and sn/snoRNA genes. To increase the number of putative $\mathrm{CB}$-associating gene loci that can be examined by microscopy, we developed a six-colour DNA FISH mapping technique to simultaneously visualize five different gene loci of interest along with coilin, the major $\mathrm{CB}$ marker protein, in cells using spectral imaging and linear unmixing (see the 'Methods' section). We confirmed co-localization of major and minor spliceosomal snRNA genes and histone genes, some of which have previously been documented to be associated with CBs individually ${ }^{22-25}$ (Fig. 1a-d). We simultaneously visualized CBs, several gene loci and four chromosome territories (CTs) which harbour major and minor spliceosomal snRNA and prominent sn/snoRNA genes. The majority of CBs were frequently located at the periphery of CTs, where two or more chromosomes interact with each other (Fig. 1b,e,f). The most frequent CTs associated with $\mathrm{CBs}$ are CT1 (62\% of CTs associated with CB) followed by CT17 (38\%), CT6 (31\%) and CT11 (31\%; Fig. 1g). After spatial analysis of the two-dimensional (2D)-projected nuclear and CT regions, we calculated the theoretical random $\mathrm{CT} 1-\mathrm{CB}$ association frequency to be $23.5 \%$. This is considerably lower than the observed frequency $(62 \%)$, indicating the specificity of this interaction and the significant role of chromosome 1 in $\mathrm{CB}$ formation. $\mathrm{CBs}$ are predominantly associated with one CT $(48 \%)$ or two CTs $(40 \%)$, and less often with three CTs (10\%; Fig. 1f). In agreement, CBs are associated with one $(46 \%)$, two $(28 \%)$ or three genomic regions $(9 \%)$ located on different chromosomes. These data indicate that $\mathrm{CBs}$ form frequent inter-chromosomal gene clusters with two or three chromosomes.

As an exception to this pattern, one $\mathrm{CB}$, and occasionally two CBs, was frequently found to be predominantly localized adjacent to chromosome 1 in HeLa cells. The uniquely identifiable CB was termed 'intra-chr1 CB'. To further investigate the role of

Figure 1 | Cajal bodies favourably interact with highly expressed $\mathbf{U}$ gene and histone gene loci. (a) Approximate chromosomal location of $B A C$ DNA FISH probe target sequences used in this study. Blue text denotes CB-proximal (4C-positive) gene loci and black text denotes non-CB-proximal (4C-negative) gene loci. (b) Six-colour fluorescent microscopy analysis of four CTs, CB-interacting gene locus detected by DNA FISH and CB localization visualized by CB marker protein, coilin, in aneuploid HeLa (CB-positive) and primary diploid D551 (CB-negative) cells. Scale bar, $2 \mu \mathrm{m}$. (c) Six-colour fluorescent microscopy analysis of Cajal body-interacting gene loci by DNA FISH and coilin immunostaining in HeLa cells. (d) Six-colour microscopy analysis of CB-interacting and CB-independent negative-control gene loci by DNA FISH and coilin immunostaining in HeLa cells. (e) Box and whisker plot of frequencies of CT-CB association interactions (manually scored by proximity to CBs). Error bars depict s.d. and scores represent assessment of at least 150 cells. (f) Histogram displaying the frequency of interaction between one CB and (up to four) CTs (manually scored by proximity to CBs). Error bars depict s.d. and scores represent assessment of at least 150 cells. (g) Box and whisker plot of frequencies of DNA FISH loci-CB association interactions (manually scored by proximity to CBs). Error bars depict s.d. and scores represent assessment of at least 150 cells. (h) Six-colour microscopy analysis of CB-interacting loci by DNA FISH and coilin immunostaining in human primary diploid foreskin D551 cells, human primary diploid cervical epithelial cells (HCVEpC; lacking CBs) and (i) HeLa cells following CB disassembly by TCAB1 or USPL1 knockdown. 
chromosome 1 in $\mathrm{CB}$ formation and function, we visualized many loci throughout chromosome 1 by six-colour DNA FISH. Several highly expressed small U RNA genes and the histone gene clusters (HIST2 and H3F3A) from different arms of the chromosome were frequently found to be associated with the intra-chr1 CB (Fig. 1c). Quantification of DNA FISH revealed that RNU1 (1p36), encoding the U1 snRNA gene array ${ }^{31}$, is the locus most frequently associated with the $\mathrm{CB}$ (Fig. 1g, median association allelic frequency $42.7 \pm 1.1 \%(N=2)$, similar to a previous report on HeLa cells $\left.{ }^{10}\right)$. Other highly expressed

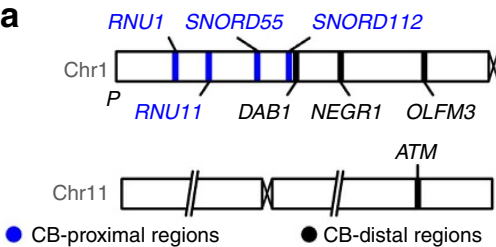

b
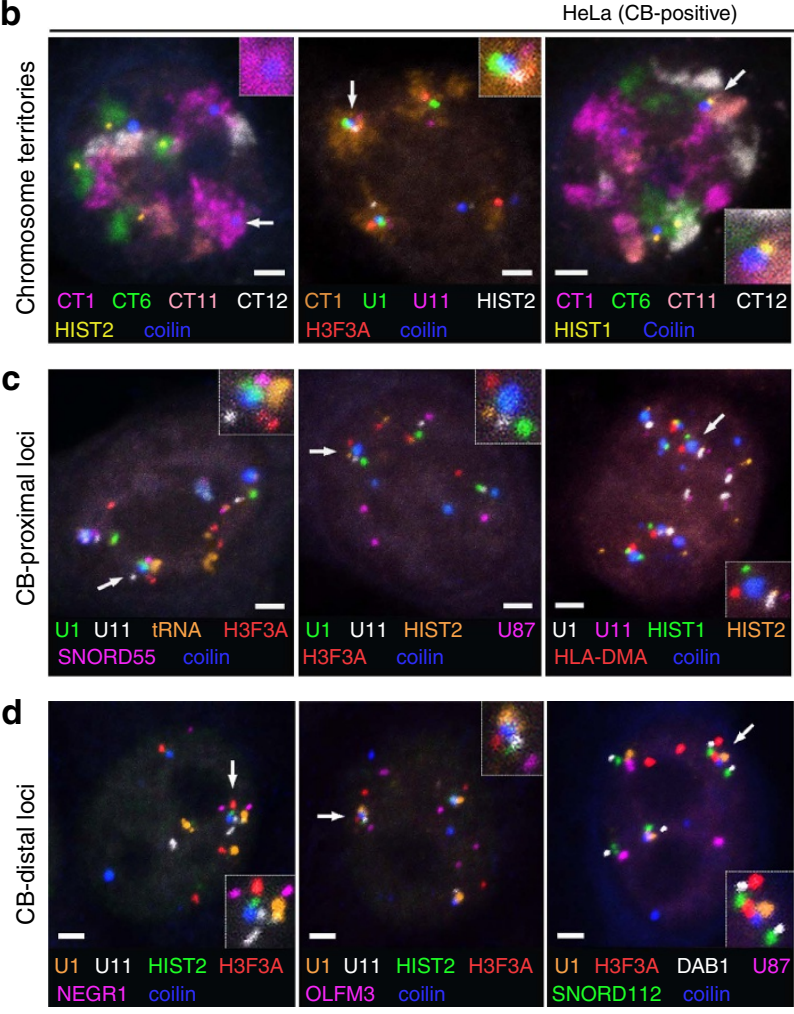

HeLa (CB-positive)
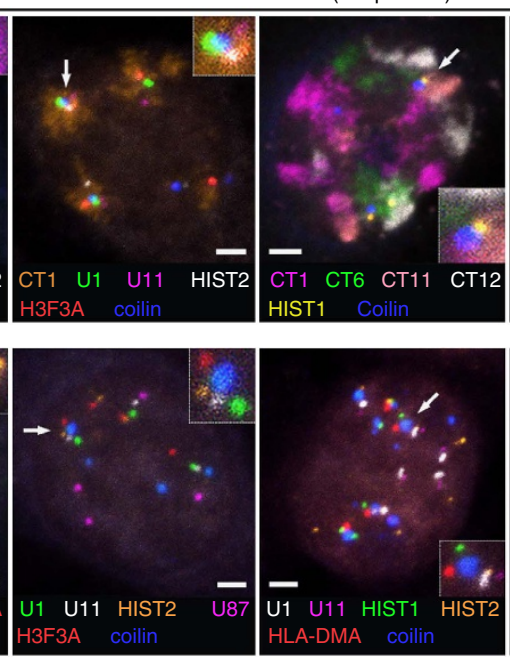

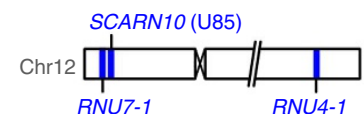

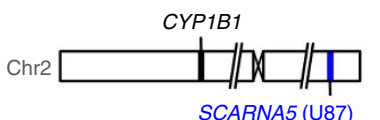

SCARNA5 (U87)
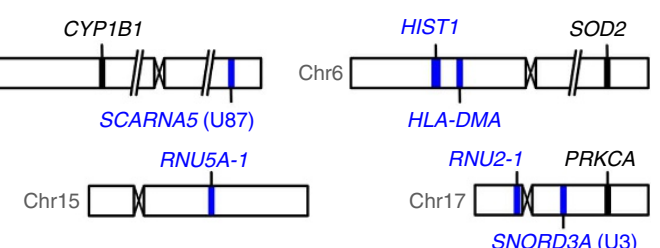

HLA-DMA

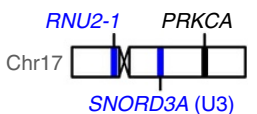

D551 (CB-negative)
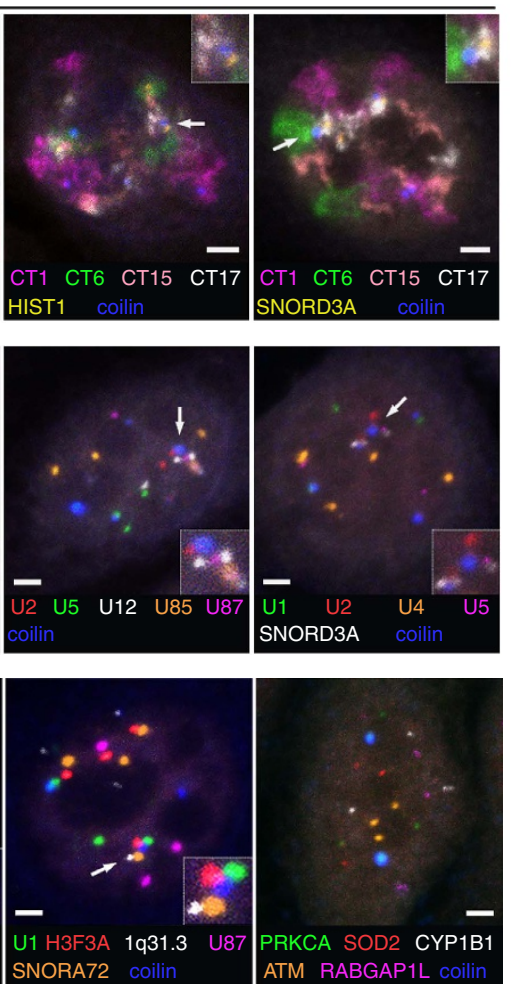
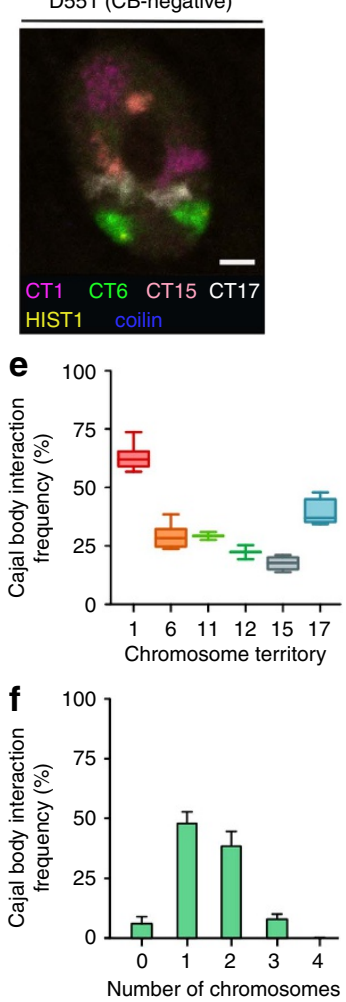

g

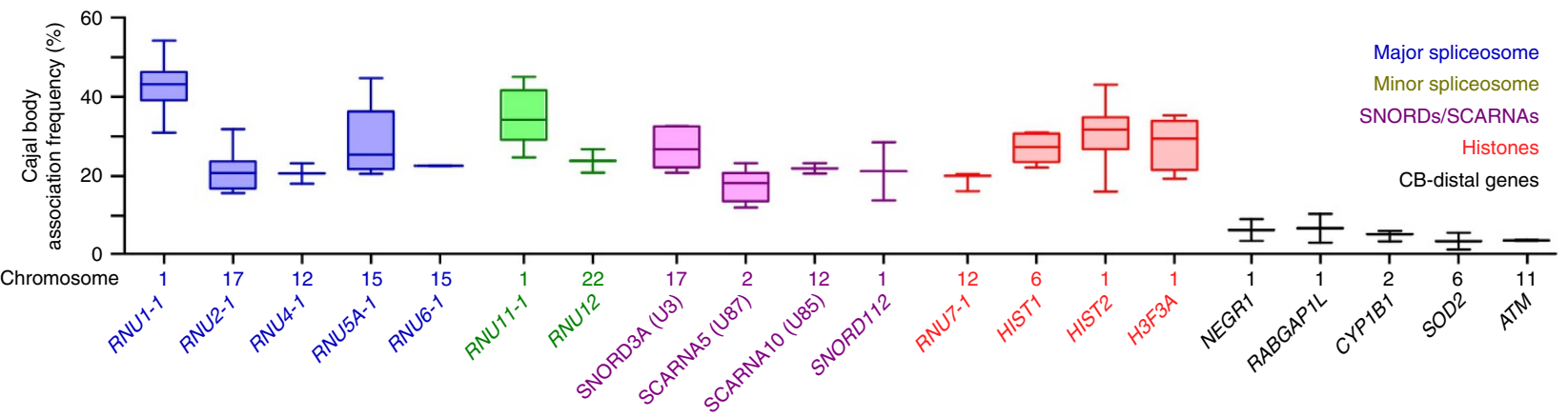

h

CB-negative

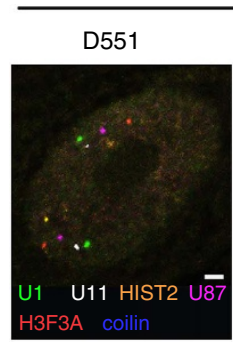

D551

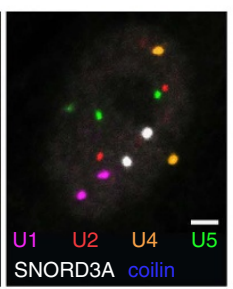

HCvEpC

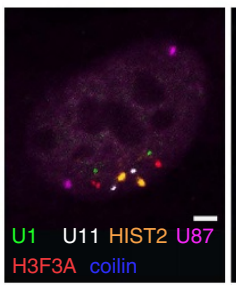

HCvEpC

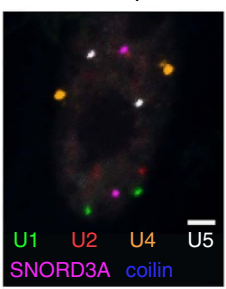

CB disassembly

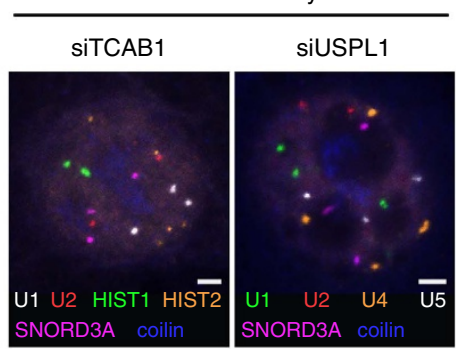


$\mathrm{U}$ snRNA and snoRNA genes residing on both arms of chromosome 1, including RNA components of the major and minor spliceosomal complexes, two histone gene clusters (HIST2 and HF3FA) and other intron-encoded genes (SNORD, SNORA and SCARNA), also display significant $\mathrm{CB}$ association frequencies (ranging from 35 to 23\%). Importantly, other genes in sn/snoRNA-poor regions, but which display expression of NEGR 1 and RABGAP1L on chromosome 1, were found to have association frequencies significantly lower (in the range of 6-8\%), suggesting that these gene-CB interactions are selective (Fig. 1d,g). We tested specific association of CBs with expressed sn/snoRNA genes by visualizing two intron-encoded snoRNA loci SNORD112, located on the p-arm (35 Mbp), and SNORA72, located on the q-arm (185 Mbp) of chromosome 1 , as well as with neighbouring $\mathrm{U}$ RNA gene-poor regions. The $\mathrm{CB}$ association frequencies of SNORD112 and SNORA72 were 21.2 and $32.4 \%$, respectively, while their neighbouring loci, $D A B 1(40 \mathrm{Mbp})$ and $1 \mathrm{q} 31.3$ (177 Mbp), had association frequencies of 9.8 and $6.1 \%$, respectively (Fig. 1a; all bp coordinates in hg19; Fig. 1g and Supplementary Fig. 1b).

Quantification of CB association of sn/snoRNA genes on other chromosomes, including SCARNA5/10 and SNORA25 (Fig. $1 \mathrm{~g}$ and Supplementary Fig. 1b), suggested that CB-U RNA gene clustering may be a genome-wide trend. The association frequency of CBs with sn/snoRNA loci on several chromosomes ranged from 12 to $20 \%$, with the exception of one genomic region on the p-arm of chromosome 17 which had a $\mathrm{CB}$ association frequency similar to RNU1 $(42.3 \pm 3.8 \%, n=2)$. This particularly high association may reflect the high-frequency simultaneous association of $\mathrm{CBs}$ with chromosome 1 and chromosome 17 (Fig. 1e). Negative control genes on other chromosomes (CYP1B1 on chr2, ATM on chr11, PRKCA on chr17) displayed an even lower association frequency with CBs than those for chromosome 1 (median 4-5\%; Fig. $1 \mathrm{~g}$ and Supplementary Fig. 1b).

Since CBs have been reported to physically associate with the HLBs, which regulate expression and the unique 3 -end premRNA processing of histone genes in a cell-cycle-dependent manner, we sought to distinguish specific histone gene clusters involved in co-clustering ${ }^{12,29}$. We co-visualized CBs together with the major and minor histone clusters, HIST1 and HIST2, and HLBs by the histone gene transcription factor NPAT ${ }^{32}$. The major histone cluster, HIST1 on chromosome 6, co-localized primarily with a HLB, whereas the minor histone cluster, HIST2 on chromosome 1, was mostly associated with a CB and in some instances with a smaller HLB (Supplementary Fig. 1d). In addition, we observed an association between another histone locus $H 3 F 3 A$, encoding the replication-independent histone variant H3.3, located on the distal end of the q-arm of chromosome 1, and RNU1 in proximity of the intra-chr1 CB (Fig. 1c,g). These data indicate that HLBs preferentially form around the major HIST1 locus and other histone loci are responsible for targeting the intra-chr1 CB where smaller HLBs form (Supplementary Fig. 1d).

CB depletion coincides with altered gene positioning. To test whether CBs actively contribute to clustering of genes, we induced the disassembly of CBs in HeLa cells by knocking down either TCAB1 or USPL1, two distinct but essential CB components. TCAB1 (also known as WRAP53 or WDR79) has been identified as a component of CB-specific scaRNAs and telomerase and is responsible for recruitment of the SMN complex to $\mathrm{CBs}^{33,34}$. SUMO isopeptidase USPL1 has also been reported as a key $\mathrm{CB}$-localized factor involved in transcription of snRNA genes ${ }^{35,36}$. When $\mathrm{CBs}$ were perturbed by siRNA knockdown of TCAB1 or USPL1, we could no longer observe the spatial clustering of previously examined sn/snoRNA loci and the histone loci (Fig. 1h). A similar pattern was observed when we analysed primary diploid cells that lack CBs (D551, Fig. 1b,h). Furthermore, we used a previously published scoring system ${ }^{37}$ to quantify the relative change in position of major histone gene cluster HIST1 within the chromosome 6 territory after CB disassembly (Supplementary Fig. 1e-h). In CB-depleted HeLa cells, the distribution of HIST1 was altered and was preferentially located within chromosome 6 (21.28 versus $48.02 \%)$ compared with the periphery of the territory (29.36 versus $16.95 \%)$ consistent with downregulation of histone genes after $\mathrm{CB}$ disassembly. These data strongly indicate that numerous sn/snoRNA and histone loci located across the genome associate with $\mathrm{CBs}$ and may require the presence of CBs to maintain their spatial organization and high expression status.

CB position mediates Chrl topology and sn/snoRNA levels. Given the evidence for CB-proximal positioning of $U$ sn/snoRNA and histone genes from DNA FISH, we sought to map the full spectrum of $\mathrm{CB}$-proximal genomic regions using the chromosome conformation capture technique ${ }^{38}$ followed by high-throughput sequencing (4C-seq) in HeLa cells (Figs 2 and 3 ). As the bait locus we selected RNU1 which exists as a highly expressed multi-copy gene array on chromosome 1 (1p36), and has the highest $\mathrm{CB}$ contact frequency among genes analysed in our six-colour DNA FISH analysis. As a complementary bait we chose the replication-independent histone gene $H 3 F 3 A$ (1q42.12) encoding the histone variant H3.3 (ref. 39) on chromosome 1, which is constitutively transcribed and frequently associated with CBs in close proximity to RNU1 (Fig. 1c). HindIII 4C libraries were constructed in replicates and sequenced to obtain the contact profiles in HeLa cells (Supplementary Fig. 3a,b). Replicate reproducibility was confirmed, and pooled $4 \mathrm{C}$ data were used for subsequent analyses (Supplementary Fig. 3c). The majority of the 4C signal indicated intra-chromosomal interactions but some

\footnotetext{
Figure 2 | Chromosome 1 undergoes a substantial topological rearrangement following Cajal body disassembly. (a) Representative 4C-seq contact profile of intra-chromosomal, CB-dependent interactions using a RNU1 bait sequence for siControl (HeLa WT, pink) and siTCAB1 (TCAB1kd, blue) treatments. A number of expressed $U$ genes were enriched within this region (dotted lines), which were determined to decrease following siTCAB1 and siUSPL1 (green) treatment by small RNA-seq. The expression of these genes within human primary cervical epithelial cells is also shown (purple). The bait region is depicted between 16.5 and $17.5 \mathrm{MBp}\left(R N U 1-2^{*}\right)$. Several genes of interest were also located in this region, denoted by arrows (orange $=$ RNA-seq expression change following TCAB1 or USPL1 knockdown). The $y$-axis displays the sequencing read counts/genome fragment, (b) representative 4C-seq contact profile of H3F3A bait to the RNU1 array region on chromosome 1 in control (pink) and siTCAB1-treated (blue) in HeLa cells. RNA-seq expression profile of RNU1-1 and RNU1-2 loci within this genomic region (dotted lines) show a sensitivity of these U genes to CB disruption by TCAB1kd or USPL1kd (green) by small RNA-seq. $y$-axis displays the read counts/genome fragment, (c) chromosome 1 RNU1 4C-seq physical contact profile (red, log10 scale) in HeLa cells under normal conditions (WT) following CB disassembly using TCAB1 siRNA. Locations of CB-proximal (blue) and -distal regions (black) used for selection of BAC probes are also indicated. (d) Validation of U RNA gene and small nucleolar RNA (SNORD/SNORA/SCARNA) expression changes in HeLa cells following siControl, siTCAB1 or siUSPL1 treatment normalized to $\beta$-actin (as depicted). $n=2,{ }^{\star} P<0.05,{ }^{\#} P<0.01,{ }^{\$} P<0.001$ compared with HeLa siControl, significance was assessed by Student's $t$-test. Error bars represent s.e.m.
} 
a
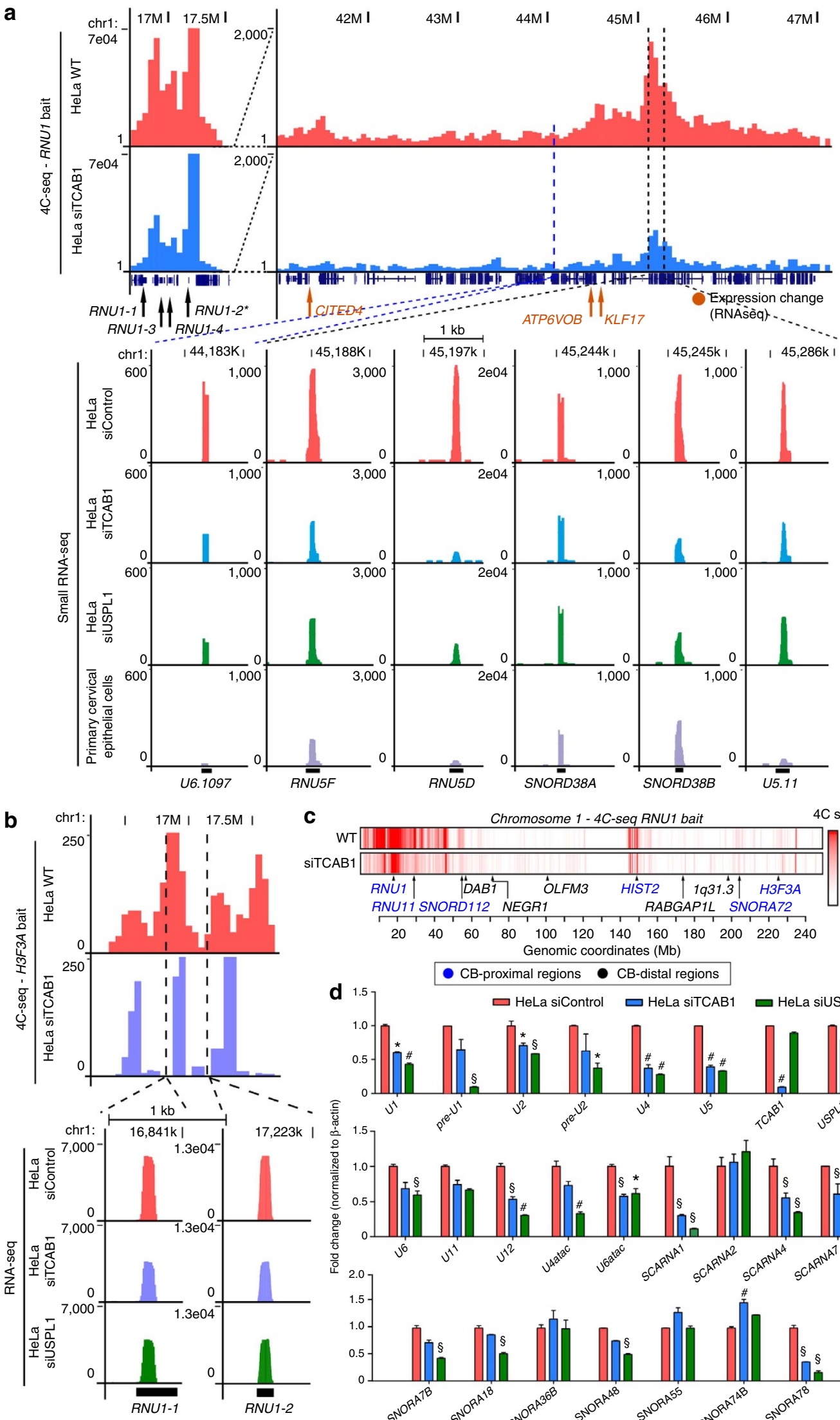

C Chromosome 1-4C-seq RNU1 bait

$4 \mathrm{C}$ signal
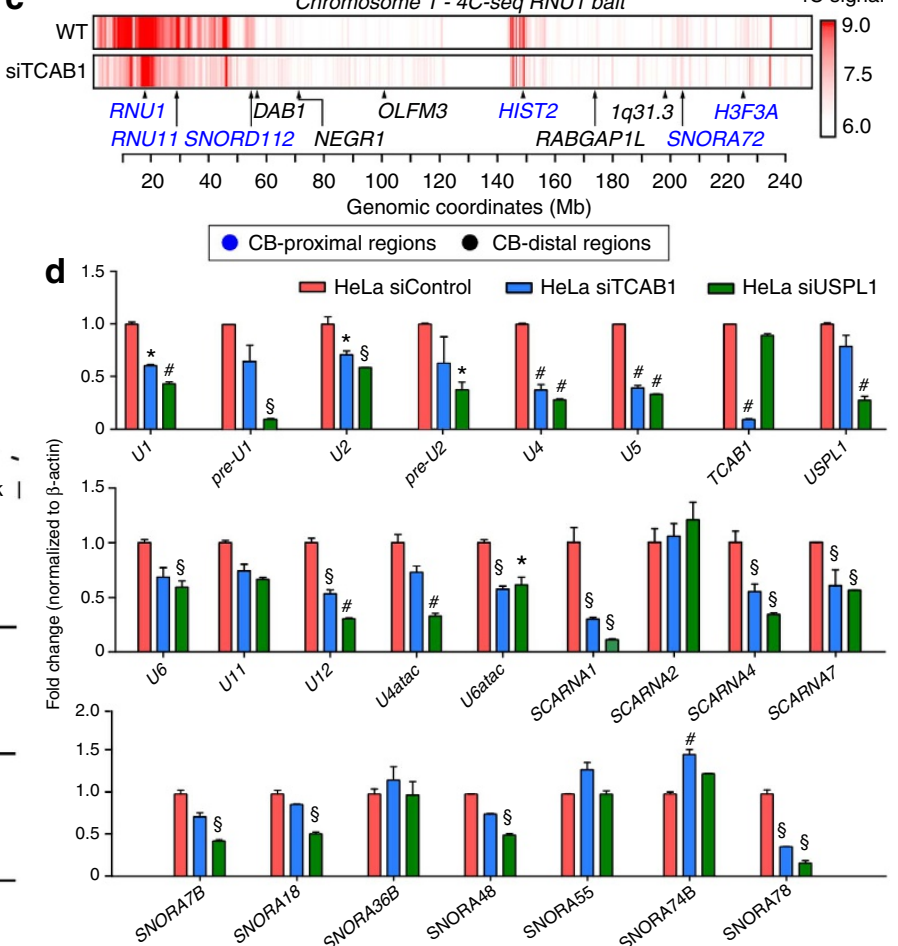
a
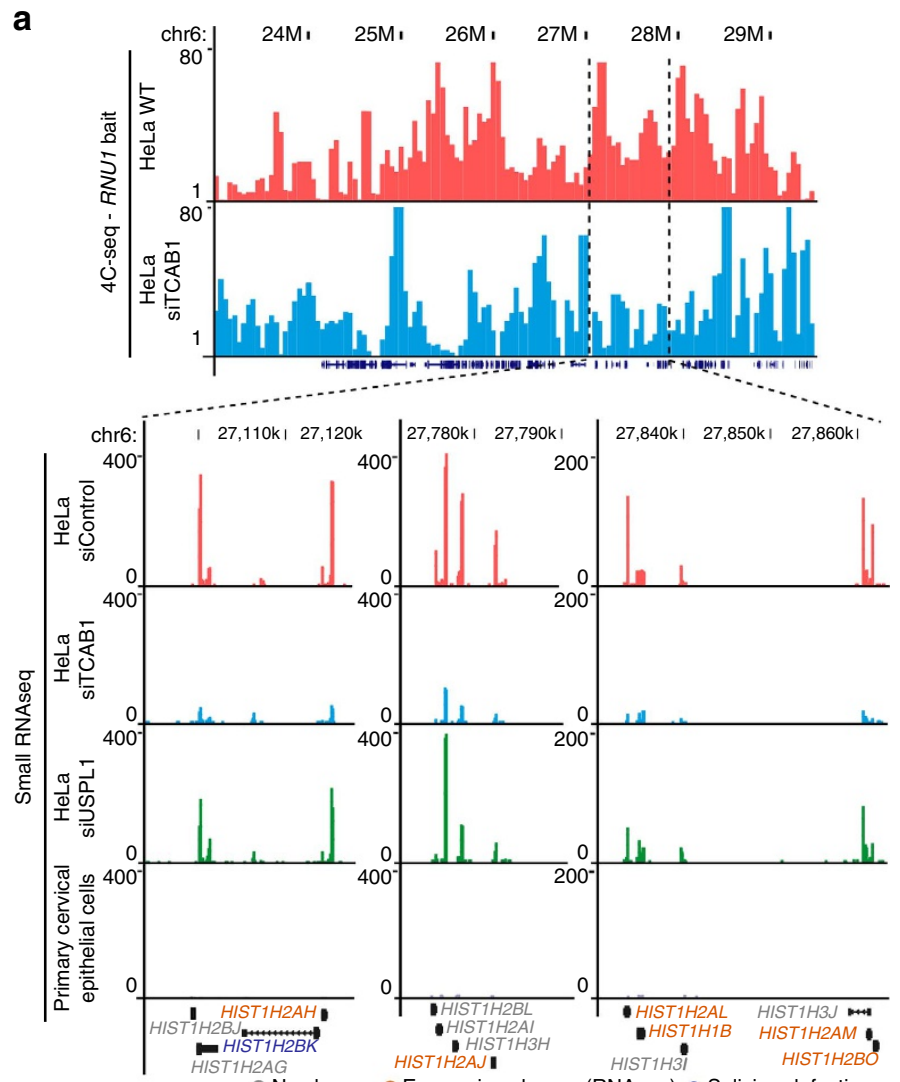

No change Expression change (RNAseq) $\bullet$ Splicing-defective genes
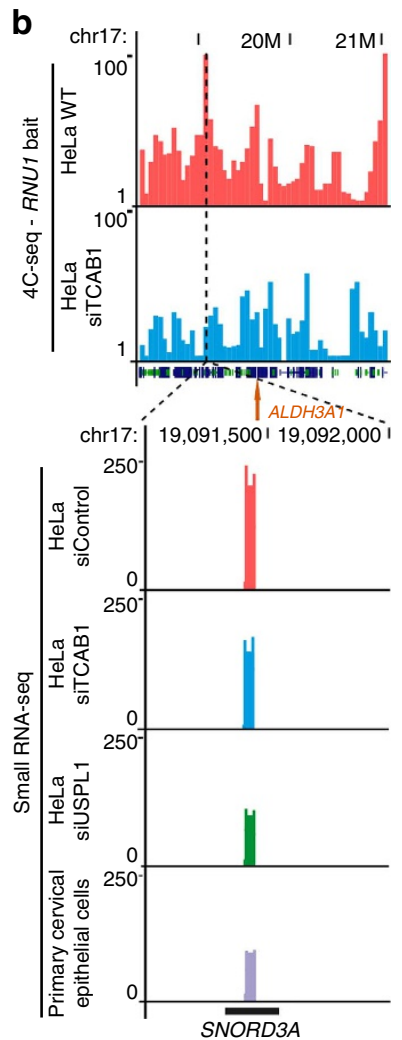

c

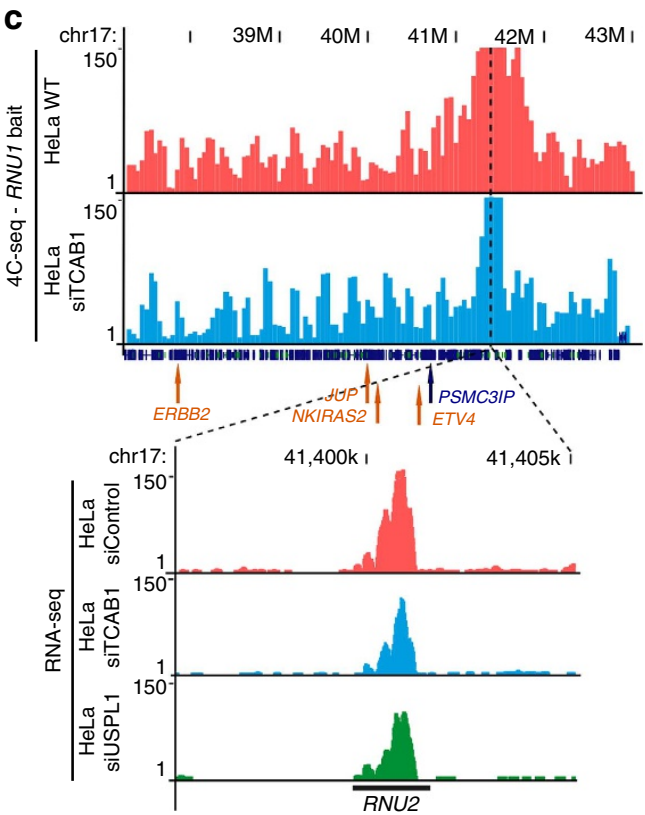

d
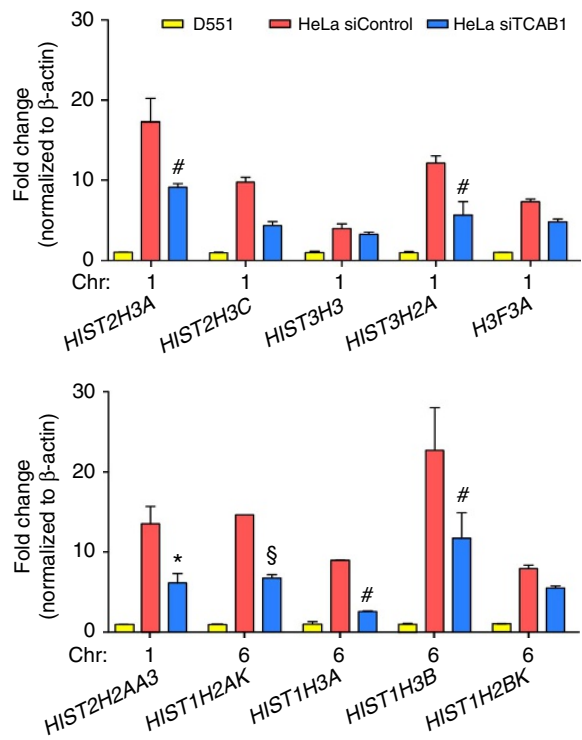

Figure 3 | CB-mediated inter-chromosomal interactions influence histone and U RNA gene expression. (a) Representative 4C-seq contact profile of inter-chromosomal, CB-dependent interactions using a RNU1 bait sequence (chromosome 1) for HeLa WT (pink) and siTCAB1 (blue) treatments at the HIST1 locus on chromosome 6. A number of expressed histone genes are enriched within this region (dotted lines) which were determined to decrease following siTCAB1 and siUSPL1 (green) treatment and poorly expressed in primary cervical epithelial cells (purple) by small RNA-seq. $y$-axis displays the read counts/genome fragment. (b,c) 4C-seq contact profile for the SNORD3A (U3 snoRNA, b) and RNU2 (U2 snRNA, c) loci on chromosome 17 using a RNU1 bait in HeLa cells. Control (HeLa WT, pink) and siTCAB1 (blue) are shown, as well as small RNA-seq profile for SNORD3A in control, siTCAB1, siUSPL1 (USPL1kd, green) and primary cervical epithelial cells (purple). $y$-axis displays the read counts/genome fragment. Several genes of interest were also located in this region, denoted by arrows (orange $=$ RNA-seq expression change following TCAB1 or USPL1kd, blue $=$ splicing defect detected, grey $=$ no change). (d) Validation of a selection of histone genes by qRT-PCR normalized to $\beta$-actin (yellow = primary D551 cells, red = HeLa siControl, blue $=$ HeLa siTCAB1). $n=2,{ }^{\star} P<0.05,{ }^{\#} P<0.01,{ }^{\$} P<0.001$ compared with HeLa siControl, significance was assessed by Student's $t$-test. Error bars represent s.e.m. 
distal inter-chromosomal interactions were also detected. The RNU1 4C profile in HeLa revealed that the contact loci interacting with the intra-Chrl $\mathrm{CB}$ through $R N U 1$ are enriched with all classes of U RNA genes (RNU, SNORD, SNORA, SCARNA, snoU and U1 snRNA class I pseudogenes on 1q12-21; $P$ value $<0.01$; see the 'Methods' section and Fig. 2a). For example, a strong interaction with RNU1 was observed for the U11 snRNA (RNU11) gene (Supplementary Fig. 2a) located $12 \mathrm{Mb}$ downstream, as well as for HIST2 and H3F3A on the other arm of chromosome 1 (Fig. 2c), consistent with the DNA FISH data.

To identify CB-dependent genome organization, we performed 4C analysis after disassembly of $\mathrm{CBs}$ using siRNA targeting TCAB1 (siTCAB1; Fig. 2, Supplementary Data 1). To understand the CB-dependent organization in terms of sn/snoRNA expression, we also performed small RNA-seq on HeLa cells treated with siTCAB1, siUSPL1 or siControl. The intra-chromosomal interactions observed in control cells were globally reduced in $\mathrm{CB}$-disrupted cells, including the chromosome end-to-end interaction between RNU1 and H3F3A (Fig. 2b,c). In parallel, a substantial fraction (11\%) of small RNA genes had a reduced expression in either knockdown (177 and 175 loci for siTCAB1 and siUSPL1, respectively), while relatively few small U RNAs showed an increased expression after knockdown (3-4\%; Supplementary Data 2). Both major and minor spliceosomal $U$ snRNA genes were downregulated following CB disassembly. The expression of these U RNA genes was typically much higher in HeLa cells (cervical carcinoma) compared with a tissue-matched control, human primary diploid cervical epithelial cells (Fig. 2a). In fact, small RNA-seq profiling revealed an astonishing range of expression for the diverse sn/snoRNA genes, spanning eight orders of magnitude. Most of the small RNA genes interacting with RNU1 belong in the highest expression category (Supplementary Data 2). We noted differential behaviours of $s n / s n o R N A s$ in CB-disrupted cells based on their status in the control primary cells. Following $\mathrm{CB}$ disassembly, the SNORA and SNORD genes that are highly expressed in the control and HeLa cells showed no major changes in expression. However, $\mathrm{U}$ sn/snoRNAs with low expression in the control cells but high in HeLa, such as the RNU1 array (RNU1-1, RNU1-2) and RNU11, had markedly decreased expression even in the same contact domain (Fig. $2 c$ and Supplementary Fig. 2a). We confirmed CB-dependent expression of sn/snoRNAs observed in small RNA-seq, including all the major and minor spliceosomal snRNA components, by qRT-PCR (Fig. 2d). Nascent extended pre-processed U1 and U2 RNA (pre-U1 and -U2) expression was decreased after $\mathrm{CB}$ disassembly indicating that $\mathrm{CBs}$ function as important transcriptional initiation sites for U RNA genes. No decreases in U RNA gene expression were observed after TCAB1kd in D551 primary diploid cells that lack CBs (Supplementary Fig. 2b). Therefore, we conclude that the observed reduction in U RNA gene expression levels is a result of CB disassembly and not secondary effects of our TCAB1 or USPL1 depletion.

Many inter-chromosomal interactions of $H I S T 1$ are CB proximal. In addition to the CB-dependent intra-chromosomal interactions of RNU1, 4C analysis indicated decreases in intra-chromosomal changes following disassembly of $\mathrm{CBs}$ (Figs 2 and 3, and Supplementary Fig. 3). Consistent with the idea that CBs may influence functional genome organization, enrichment for sn/snoRNA genes within contact regions from both RNU1 and H3F3A 4C was reduced in the absence of CBs (permutation test; see the 'Methods' section). Although detection of interchromosomal contact dynamics is technically challenging with
4C, interaction of RNU2 (ref. 40) and SNORD3A (U3), both on chromosome 17, with RNU1 depended on CBs (Fig. 3b,c), and their expression was notably diminished in CB-disrupted cells.

Histone gene clusters on chromosomes 1 and 6 also had CB-dependent interactions with both RNU1 and H3F3A loci. In particular, HIST1 on chromosome 6 contains over fifty replication-dependent histone genes. Similar to sn/snoRNAs, the expression of HIST1 genes, highly abundant in HeLa cells, was suppressed after CB disassembly (Fig. 3a,d). This can likely be ascribed to the concomitant loss of the association of HLBs with disassembled CBs. These results suggest a role of CBs in genome organization that reaches beyond chromosome 1, promoting the physical clustering of sn/snoRNAs and histone loci and their robust expression.

High-content DNA FISH CB co-localization analysis. Interactions captured by $4 \mathrm{C}$ rely on high contact frequency between RNU1 and the intra-chr1 CB and do not directly establish the interactions between $\mathrm{CB}$ and the RNU1 contact loci ${ }^{41}$. To quantitatively assess how often RNU1 cis-contacts are co-localized with the intra-chr1 $\mathrm{CB}$, we used automated high-throughput microscopy of DNA FISH/IF co-localization patterns (see the 'Methods' section and Fig. 4). Following data acquisition from $>1,000$ cells per locus pair and automated nuclear segmentation and spot detection of DNA FISH and IF signals, we analysed the minimum pairwise Euclidean distances and classified co-localizations based on whether they occur near a $\mathrm{CB}$ (Fig. 4b). RNU1 displayed a preferential association with CBs, confirming our earlier DNA FISH data (Fig. 4c). When several 4C loci were probed along chromosome 1, RNU1 contact frequency for all of the 4C-positive loci was significantly higher compared with the 4C-negative control locus located on the same chromosome arm (Fig. 4d). Our high-throughput approach detected some of the gene co-localizations involving RNU1 occurring away from CBs (Fig. 4f, 'CB-distal gene interaction'), which likely correspond to stochastic predecessors of CB-associated gene clusters. These interactions occurred as frequently as gene-pairing events between NEGR1 and other sn/snoRNA-poor regions. Importantly, the RNU1 association frequency profile over all the examined loci was independent of whether they were proximal to $\mathrm{CBs}$ or not (Fig. 4f and Supplementary Fig. 4a,b) and greater than the CB-proximal pairing frequency displayed by NEGR1 and other sn/snoRNApoor loci (Fig. 4g, Supplementary Fig. 4c). Disassembly of CBs by TCAB1kd resulted reduced the association frequency between RNU1 and SNORD112 (two CB-proximal gene loci) but increased the frequency of gene pairing between RNU1 and RABGAP1L (sn/snoRNA-deficient) confirming our hypothesis that CBs are an important genome organizer in HeLa cells (Fig. 4h). These phenomena were not due to previously reported changes in cell cycle progression, ${ }^{42}$ as gene pairing between $R N U 1$ and other sn/snoRNA-dependent regions, but not a sn/snoRNA-poor region, was increased on induction of a cell cycle block by double thymidine at the G1/S border (Fig. 4i). In addition, expression of a number of highly sensitive $U$ RNA and sn/snoRNA genes was largely unaffected by cell cycle perturbation (Supplementary Fig. 4f)

CB depletion induces limited global transcriptional effects. To examine the role of $\mathrm{CBs}$ in transcription, we analysed the expression profiles from RNA-seq performed on CB-depleted HeLa cells using siRNA-mediated knockdown of TCAB1 or USPL1. We found that gene expression profiles in both knockdowns and siControl HeLa were similar $(r=0.99)$, indicating that the global effects of $\mathrm{CB}$ disassembly on 
a

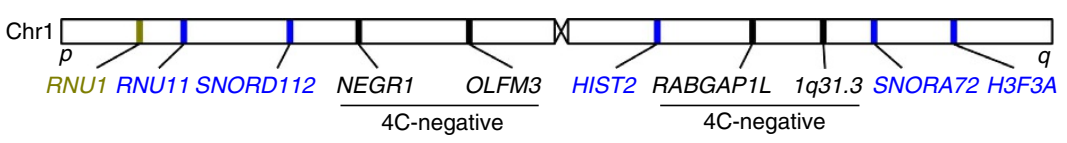

b
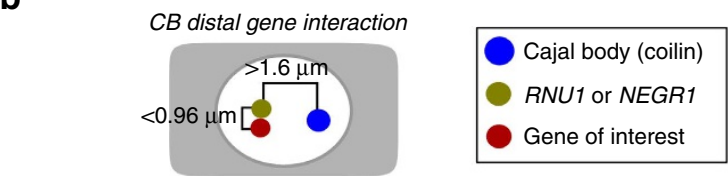

CB proximal gene interaction

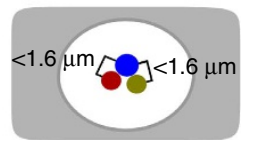

C

d
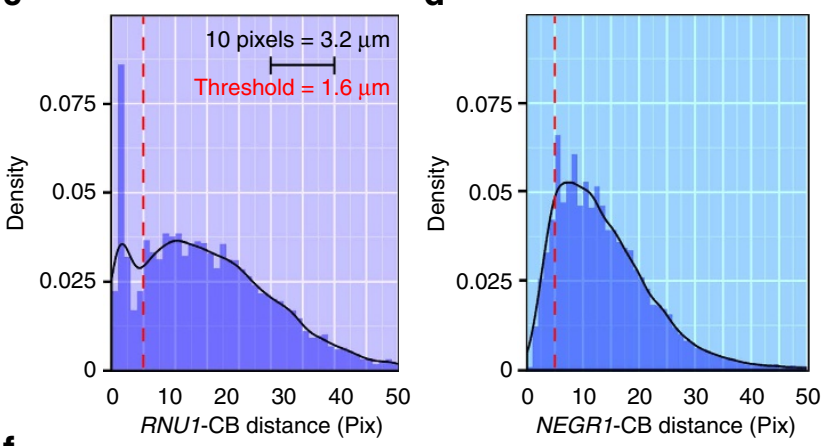

e

$\mathbf{f}$

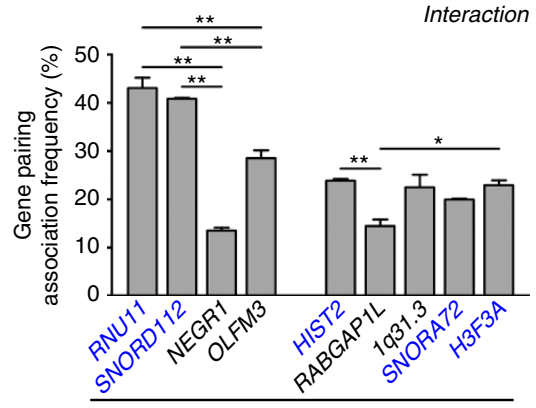

CB distal gene interaction

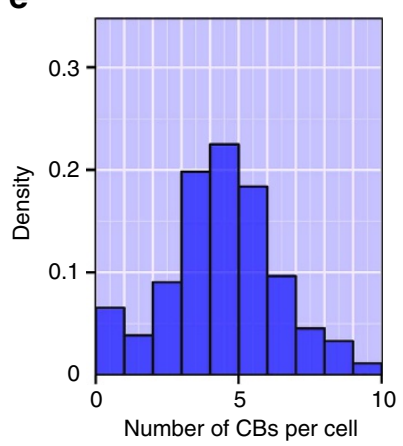

Interaction with RNU1

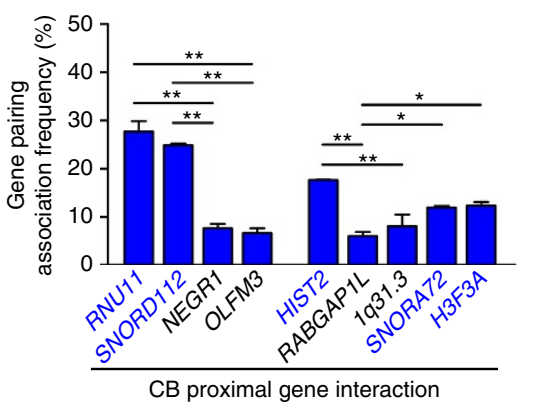

Interaction with NEGR1
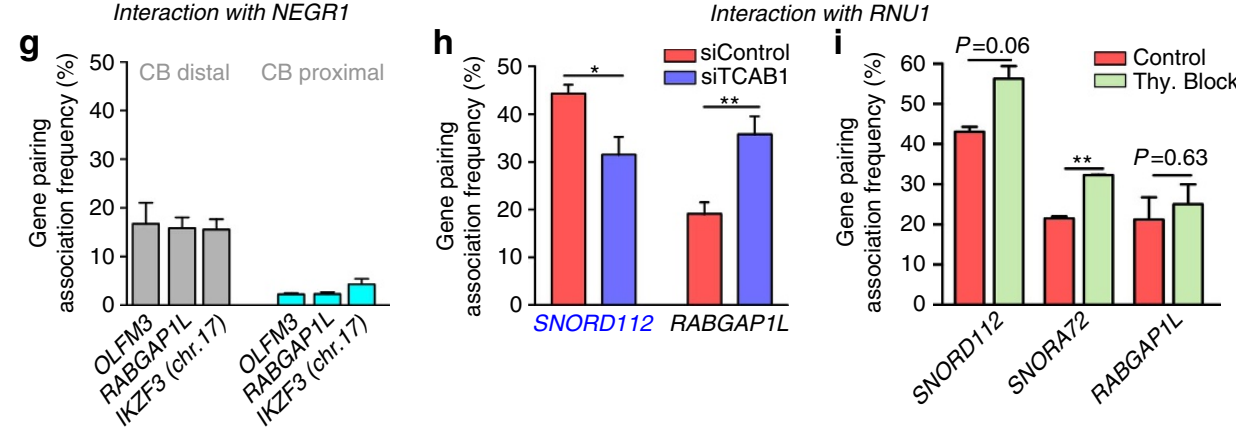

Figure 4 | High-content microscopic validation of Cajal body-dependent gene interactions. (a) Approximate chromosomal location of BAC DNA FISH probe target sequences used in automated high-throughput DNA FISH-IF co-localization studies. Blue text denotes CB-interacting (4C-positive) loci. Regions determined to be non-CB interacting are denoted by ( $4 C$-negative, black text). (b) A schematic detailing the threshold criteria used to determine whether a gene-gene cis-interaction or gene-CB interaction was meaningful. The RNU1 gene and various gene locus of interest located on chromosome 1 were determined to interact in the absence of $C B s$ when distance was $>5$ pixels from a $C B$, but $\leq 3$ pixels between spot centres (CB-independent). CB-clustered gene loci were determined to occur within a distance of $\leq 5$ pixels between the CB spot centre and the RNU1 loci spot centre (CB-dependent). Red spot $=R N U 1$, green $=$ variable gene of interest, purple $=\mathrm{CB}$. (c) Histogram of the per-RNU1 FISH spot minimum distances between RNU1 and CB (in pixels, 10 pixels $=3.2 \mu \mathrm{m})$. Blue bars = binned data, black line $=$ estimated density function, red dotted line $=$ threshold criteria $(1.6 \mu \mathrm{m})$. $(\mathbf{d})$ Histogram of the per-CB spot minimum distances between NEGR1 and CB (in pixels, 10 pixels $=3.2 \mu \mathrm{m}$ ). Blue bars = binned data, black line $=$ estimated density function, red dotted line $=$ threshold criteria $(1.6 \mu \mathrm{m})$. (e) Histogram depicting the number of CBs per cell in HeLa cells as quantified by high-throughput imaging ( $>1,000$ cells). (f) Association frequency between RNU1 and various genes of interest on chromosome 1 (plotted in order relative to RNU1) as determined by automated high-throughput imaging. Interactions were grouped according to the criteria detailed in $\mathbf{b}$. ( $\mathbf{g}$ ) Comparisons were also made between sn/snoRNA-poor loci. Total gene pairing was also assessed after (h) siTCAB1 treatment and (i) cell cycle arrest by double-thymidine treatment (Thy. Block). $n=2$ ( $>1,000$ cells per replicate), error bars depict s.e.m., ${ }^{\star} P<0.05,{ }^{\star \star} P<0.01,{ }^{\star \star \star} P<0.001$ versus $R$ NU1-NEGR1 or RNU1-RABGAP1L was assessed by Student's $t$-test. Error bars represent s.e.m. CB-distal interactions $=$ grey, CB-dependent interactions $=$ dark blue. Red $=s i C o n t r o l$, blue $=s i T C A B 1$, light green $=$ double-thymidine block. 
a

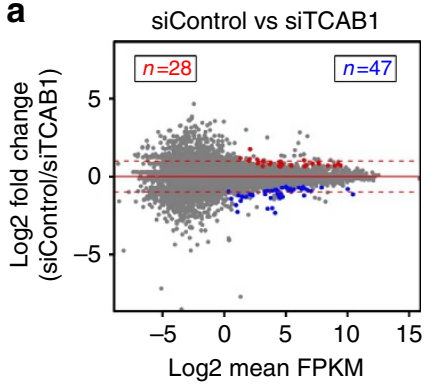

b
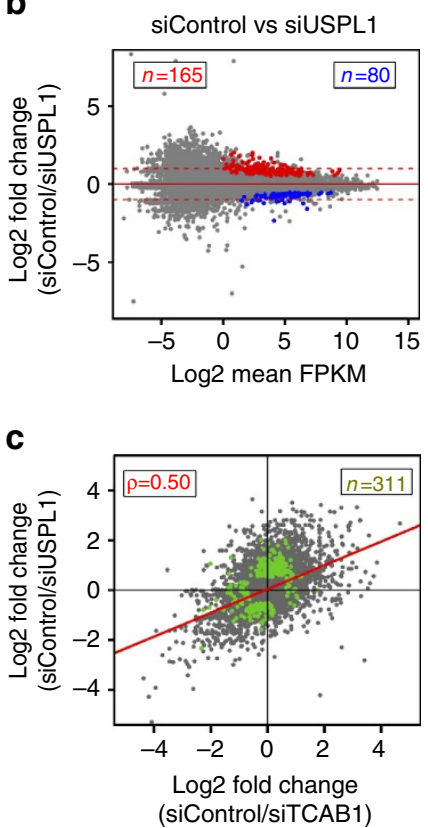

d

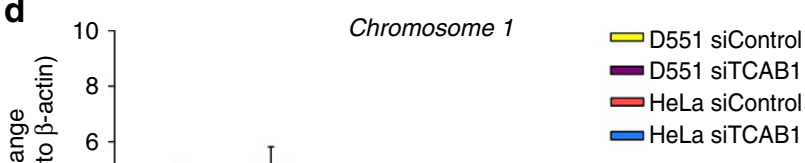

HeLa siControl
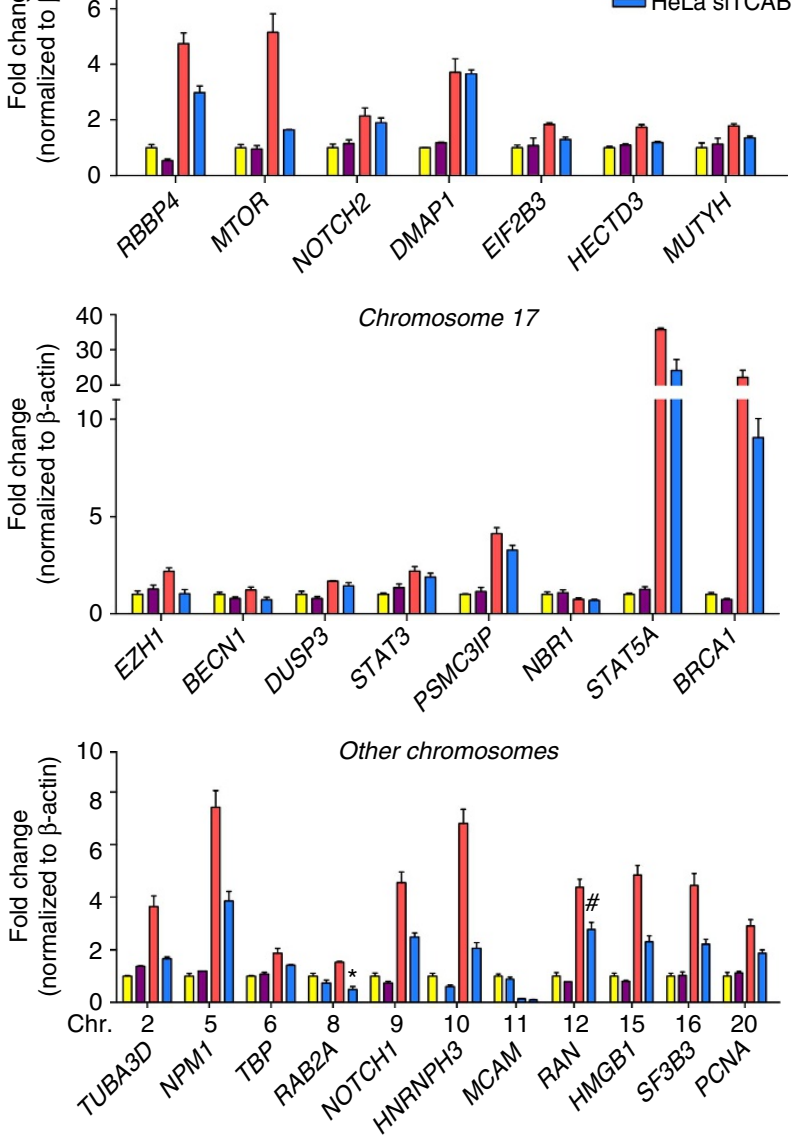

Figure 5 | Differential gene-level transcription and splicing following Cajal body disruption. (a,b) Ratio versus mean abundance scatterplots for the siTCAB1 (a) and USPL1 (b) treatments. Coloured dots represent gene differentially expressed (at FDR-adjusted $P$ value $<0.01$, red $=$ higher in siControl, blue $=$ higher in knockdown). (c) Ratio/Ratio scatterplot of fold-change differences in either siUSPL1 or siTCAB1 treatments. Dots in green have adjusted $P$ value $<0.01$ in either comparison. Dots in the lower left and upper right quadrants indicate fold changes in the same direction. (d) Validation of a selection of genes within $4 C$ contact regions by qRT-PCR normalized to $\beta$-actin (yellow = primary D551 cells, red $=$ HeLa siControl, blue $=$ HeLa siTCAB1). $n=2$, ${ }^{\star} P<0.05,{ }^{\#} P<0.01,{ }^{\$} P<0.001$ compared with HeLa siControl, significance was assessed by Student's $t$-test. Error bars represent s.e.m.

transcription are modest (Fig. 5a-c). We confirmed the RNA-seq results by $\mathrm{qRT}-\mathrm{PCR}$, validating that $\mathrm{CB}$ disassembly induces various but limited expression changes of other Pol II-dependent genes within 4C contact regions (Fig. 5d). Despite the requirement for $\mathrm{CBs}$ in sn/snoRNA gene transcription, we found no significant transcriptional changes of host genes whose introns encode $\mathrm{U}$ snoRNAs in $4 \mathrm{C}$ contact regions. However, we identified 259 genes, showing differential expression in knockdown versus siControl (Supplementary Data 3), and intersected these genes with RNU1 and H3F3A 4C data. We found that only 87 genes (34\%) residing within $50 \mathrm{~kb}$ of $4 \mathrm{C}$-positive contact regions, and the majority $(66 \%)$ of the mis-regulated genes were farther away, suggesting that the transcriptional effect reaches beyond the genes in immediate physical proximity of CBs. Among the genes within $50 \mathrm{~kb}$ of $4 \mathrm{C}$ contact regions, several involved in splicing (for example, SNRPA1, PRPF3, SMC1A, GEMIN5) were differentially expressed. In addition, multiple histone genes from histone cluster 1 (HIST1) and 2 (HIST2) displayed expression changes. A summary of CB-dependent genes and their ontology is shown in Supplementary Fig. 6.

Together with the small RNA-seq data indicating that the disassembly of CBs induces a significant curtailment of U RNA gene expression, the $\mathrm{CB}$ also contributes to high expression levels of key spliceosomal components, such as SNRPE (Sm protein E) and SNRPA (U1 snRNP-specific protein A) but may also be required for their sustained expression. This is in accordance with the role of CBs as a catalytic processing site for spliceosome reassembly.

We also observed potential feedback control mechanisms between $\mathrm{CB}$ components. $\mathrm{CB}$ disruption by specific knock-down conditions invoked compensatory upregulation of COIL, the gene encoding coilin, the critical self-interacting structural $\mathrm{CB}$ component, and Gemin5, another CB component of the SMN protein complex. In contrast, the gene encoding the Integrator subunit S3 (a member of the Integrator complex responsible for the $3^{\prime}$-end processing of nascent extended pre-U snRNA transcript $\left.{ }^{43,44}\right)$, INTS3, which directly interacts with coilin and is able to nucleate CBs de novo, is downregulated.

CB disassembly coincides with altered RNA processing. To assess whether RNA processing is perturbed after $\mathrm{CB}$ disassembly, we examined splicing patterns in the total RNA-seq data. Using an exon-centric approach (DEXSeq $\left.{ }^{45}\right)$, we identified 
40 genes with differential exon expression in knock-down samples (Supplementary Data 4). The most enriched gene ontology category in this gene set was chromatin organization. $50 \%$ of these differentially expressed exons reside within $50 \mathrm{~kb}$ of $4 \mathrm{C}$ contact regions. Next, we performed a junction level analysis with a comprehensive classification of pairwise splicing events (Supplementary Data 5). A genome-wide summary of the RNAseq and small RNA-seq data is presented in Supplementary Fig. 5. We identified 1,050 differentially spliced events $(q$-value $<0.01$, Fig. $6 \mathrm{a}-\mathrm{c})$. The effects in the two knockdowns were generally in the same direction $(r=0.66$, Fig. $6 \mathrm{c})$, supporting that TCAB1 and USPL1 are operating in similar pathways. We performed a meta-analysis of these two approaches with stringent criteria, and identified 85 genes with high confidence showing differences in their splicing (Supplementary Data 5). We found that a substantial proportion (42\%) of these genes reside within $50 \mathrm{~kb}$ of $4 \mathrm{C}$ contact regions. Among the events differentially detected is an exon skipping event in THOC5, encoding a protein associated with the spliceosome. For another example, $B U B 3$, involved in cell-cycle progression, shows a minor alternative splicing acceptor site used less frequently after TCAB1 or USPL1 knockdown (Fig. 6d,e).

Since splice junction analysis relies on gene annotations that are incomplete, we examined whether unannotated splice junctions are present in each sample, after adjusting for sequencing depth and transcription. These splice junctions may represent aberrant splicing events resulting from a loss of splicing fidelity. There were 11,515 unannotated junctions uniquely detected in siControl samples, indicating an inherent level of splicing noise in HeLa cells. Interestingly, we detected 30,132 unannotated splice junctions in siTCAB1 or siUSPL1 samples that were absent in siControl samples (Supplementary Data 5, Fig. 6f). The apparent lack of splicing fidelity was also observed at the gene level. These junctions belong to 9,077 genes, among which $6,424(71 \%)$ had more than one unannotated junction. For example, five unannotated junctions for SNRPE were detected in the CB-disrupted condition but undetectable in the control HeLa. Fifteen genes with complex annotated splicing each had at least 20 unannotated junctions. Among these, the number of unannotated junctions within $A D A R B 1$, increased from 8 in the control to 31 in CB-disrupted cells. This gene encodes a protein responsible for adenosine-to-inosine RNA editing ${ }^{46}$, which raises the possibility of another layer of post-transcriptional regulation influenced by $\mathrm{CBs}$. Furthermore, the majority of genes within $\mathrm{CB}$ contact regions had at least one unannotated junction after $\mathrm{CB}$ disassembly ( 72 and $83 \%$ of genes within $25 \mathrm{~kb}$ of the RNU1 and H3F3A 4C regions, respectively). However, the enrichment was not statistically significant when we considered genes of comparable expression status within the 4C-negative regions $(70 \%)$, indicating that the altered splicing pattern reaches beyond the CB-proximal genes. There was minimal enrichment of small $\mathrm{U}$ RNAs encoded in the introns of abundant host genes which were differentially spliced after $\mathrm{CB}$ disassembly compared with all genes $(4.1 \%$ versus $9 \%, P=0.025$, Fisher's Exact Test) but this was not replicated in our unannotated splicing analysis ( $1 \%$ of junctions coincide with a small RNA gene). Thus, the broad post-disassembly of CBs increase of aberrant junctions suggests that $\mathrm{CBs}$ promote splicing fidelity, as well as efficiency of splicing.

We also observed additional effects that could not be directly attributed to splicing. The largest subunit of RNA polymerase (POLR2A) contains an exon differentially expressed in siTCAB1 samples. Notably, this is the last exon of the gene encoding the CTD tail, which is critical for the polymerase function and facilitates capping and splicing ${ }^{47}$ (Fig. 6h,i). Due to its location within the gene body, alternative polyadenylation is the most likely underlying mechanism. We confirmed these RNA-seq findings by qRT-PCR, including decreased expression of the Pol II CTD tail, as well as for several other genes (Fig. 6g,j). The effect on Pol II CTD suggests the possibility that some of the transcriptional changes in CB-disrupted cells observed away from RNU1 contact regions may arise indirectly from the altered form and decreased level of RNA polymerase subunits.

The above phenomena regarding mRNA processing after $\mathrm{CB}$ disassembly are consistent with the highly reduced expression of many spliceosomal U RNA genes in CB-disrupted cells observed from the small RNA-seq data. Alternatively, reduced expression of histone genes has also recently been linked to decreased co-transcriptional splicing and increased splicing noise. ${ }^{48} \mathrm{We}$ note that the splicing noise was elevated after $\mathrm{CB}$ disruption using two independent methods of disassembling CBs, through TCAB1 and USPL1.

\section{Discussion}

$\mathrm{CB}$ formation is thought to involve stochastic self-organization, initiated by nucleating factors coalescing and accumulating at distinct transcriptionally active nuclear sites. We have previously documented that $\mathrm{CBs}$ and physically associated HLBs can be formed de novo by tethering unprocessed histone pre-mRNAs or specific CB components on a gene locus which then act as seeding elements ${ }^{12,49}$. Live-cell imaging studies have shown that CBs can go through fusion and fission process as the cell cycle progresses ${ }^{50}$. Highly active transcription may also promote the specific gene locus association with $\mathrm{CBs}$, as evidenced by an engineered inducible U2 snRNA gene array targeting a $\mathrm{CB}$ in

Figure 6 | CB depletion increases unannotated RNA splicing including RNA polymerase II CTD deletion. (a,b) Difference in percentage spliced in (Delta PSI) versus mean abundance (log10 average total read counts) scatterplots for siTCAB1 (a) and siUSPL1 (b) treatments. Coloured dots represent local pairwise splicing events that are differentially spliced (at FDR-adjusted $P$ value $<0.01$, red $=$ higher in siControl, blue $=$ higher in knockdown). (c) Delta PSI/ Delta PSI scatterplot of splicing differences in siUSPL1 or siTCAB1 treatments. Dots in green have adjusted $P$ value $=0.01$ in either comparison. Dots in the lower left and upper right quadrants indicate fold changes in the same direction. (d) Sashimi plot of RNA-seq coverage over exons and junctions in the BUB3 locus. Differential splicing of an alternative acceptor is indicated. Squares are mosaic plots of splice junction counts within this splicing event.

(e) Validation of alternative splicing of BUB3 detected by DEXseq through qRT-PCR in HeLa cells treated with siControl (red), siTCAB1 (blue) or siUSPL1 (green). Primers detected exon 6, 8, 9, $7+8$ or/and +9 (as depicted). $n=2$, significance was assessed by Student's $t$-test. Error bars represent s.e.m. (f) Distribution of splice junctions detected in HeLa cells exposed to siControl, siTCAB1 or siUSPL1 (blue, red and green, respectively). (g) Validation of alternative splicing of a selection of genes detected by DEXseq through qRT-PCR in HeLa cells treated with siControl (red), siTCAB1 (blue) or siUSPL1 (green). $n=2,{ }^{\star} P<0.05,{ }^{\#} P<0.01, \$ P<0.001$ compared with HeLa siControl, significance was assessed by Student's t-test. Error bars represent s.e.m. (h) Location of primer sequences recognizing exons 5, 22 (both non-CTD) and 29 (CTD tail) within the gene encoding the largest subunit of RNA polymerase II (POL2RA), which were used to validate a previously unannotated alternative splicing mechanism for POL2RA. (i) DEXseq results for the POL2RA locus, indicating significant differential expression of exon 29, encoding the CTD tail under TCAB1 (blue) or USPL1 (green) knock-down conditions. (j) Validation of differential exon usage of POL2RA detected by DEXseq through GRT-PCR, using primers briefly detailed in $\mathbf{h}$, in HeLa cells treated with siControl (red), siTCAB1 (blue) or siUSPL1 (green). $n=2$, error bars represent s.e.m. 
proximity by an actin-dependent long-range movement after transcriptional activation ${ }^{37}$. Our current study shows that small $\mathrm{U}$ RNA genes of all categories across the genome are capable of forming specific intra- and inter-chromosomal gene clusters around CBs, consistent with a recent coilin ChIP-seq study that identified several U RNA gene loci as potential coilin-interacting sites $^{51}$. Since the early gene interactions presumably occur stochastically before the formation of structurally stable $\mathrm{CBs}$, the highly active tandemly repeated gene arrays such as $R N U 1$, RNU2, and histone gene clusters may eventually coalesce with other transcriptionally active sn/snoRNA genes on other chromosomes around CBs. Our RNA-seq analysis also revealed an additional layer of self-regulation for $\mathrm{CB}$ maintenance. Disruption of CBs by specific knockdown of component factors

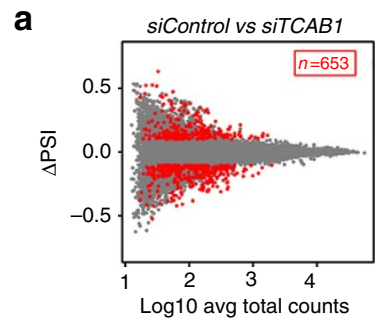

d

b
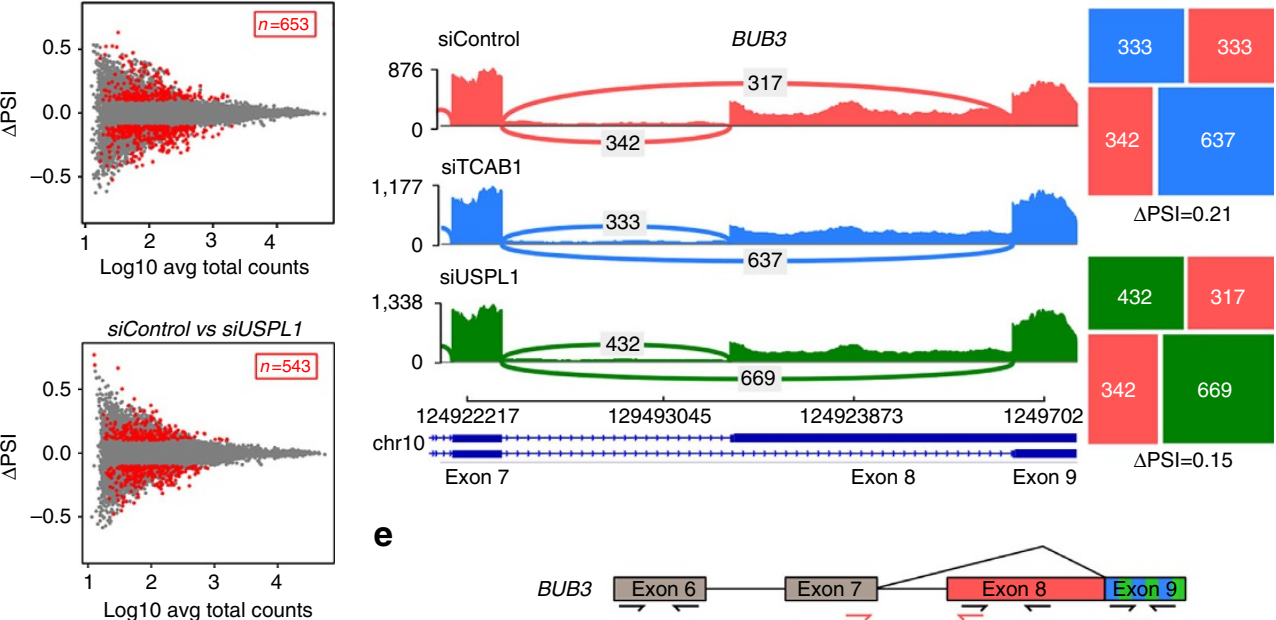

C

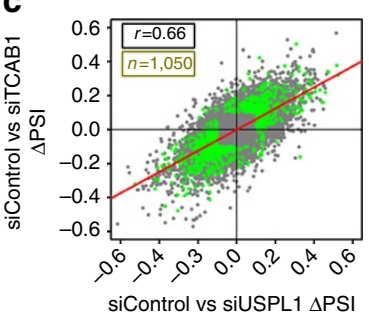

e

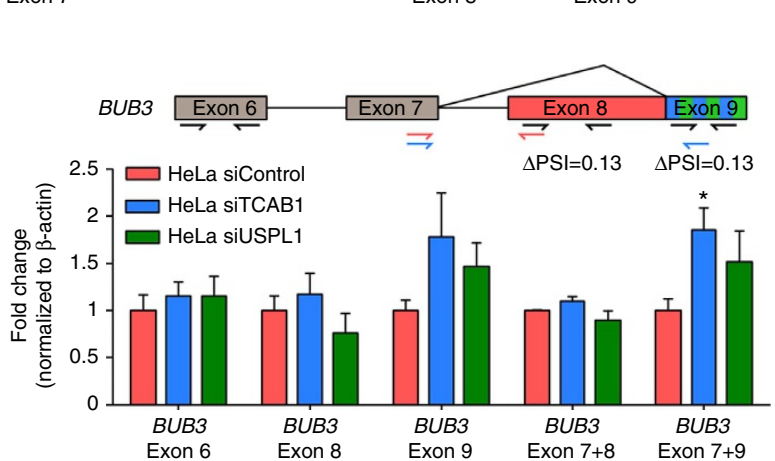

$\mathbf{f}$

Unannotated splice juncitons detected in each sample

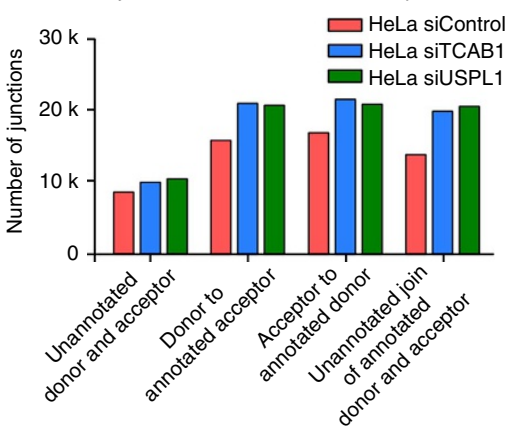

g

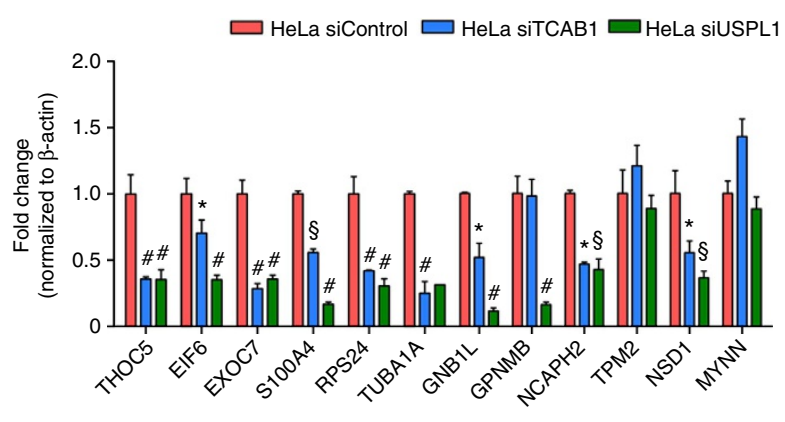

h

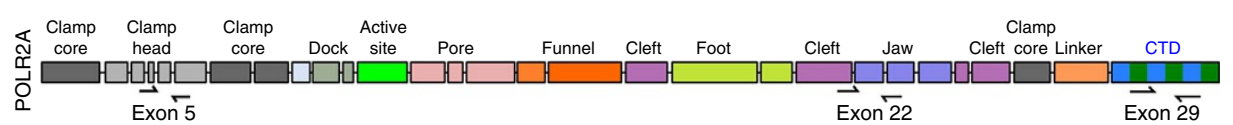

i

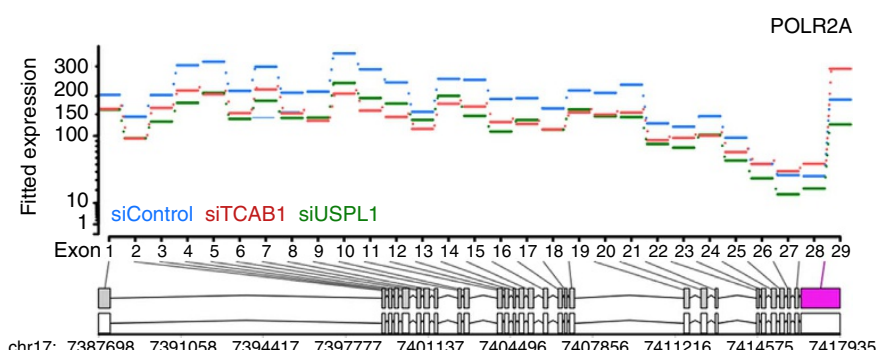

chr17: $\begin{array}{llllllllll}7387698 & 7391058 & 7394417 & 7397777 & 7401137 & 7404496 & 7407856 & 7411216 & 7414575 & 7417935\end{array}$

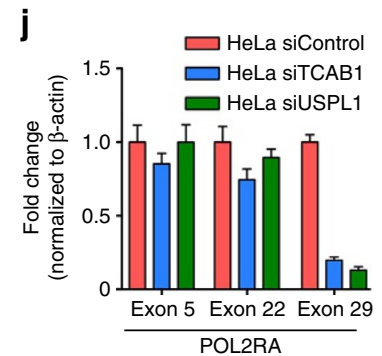


invoked a feedback control mechanism to compensate for the loss of CB structure, including upregulation of critical CB components (coilin and Gemin5) and downregulation of a CB-interacting factor of the Integrator complex (INTS3) which regulates pre-U RNA 3'-end processing ${ }^{43,44}$. Further work will be necessary to understand the selectivity of such regulation.

Multiple complementary approaches were used in our study to demonstrate that chromosome 1 may play a potentially indispensable role in the nucleation of CBs. Chromosome 1 adopts a topological arrangement around the intrachromosomally positioned $\mathrm{CB}$, frequently juxtaposing the two opposite chromosomal ends in close proximity. The physical proximity of a vast majority of highly expressed small $U$ RNAs and histone genes on chromosome 1 to the $\mathrm{CB}$ may create a spatial configuration that accelerates the processing of accumulated or stalled nascent transcripts from these loci.

Since CBs are often physically associated with $\mathrm{HLBs}^{12,29}$, we analysed their relationships and were able to distinguish which specific histone gene clusters contribute to their formation. Our six-colour DNA FISH revealed that the large major histone gene cluster HIST1 on chromosome 6 primarily nucleates CB-protruding HLBs, and the smaller minor histone gene cluster HIST2 on chromosome 1 is mostly associated with the $\mathrm{CB}$ and a smaller HLB. These data support the distinct roles of different histone gene clusters on the two chromosomes.

Through a combination of 4C-seq, total RNA-seq, small RNA-seq and qRT-PCR analysis, we characterized the genome-wide impact of CBs on chromosome architecture and $\mathrm{CB}$-dependent gene regulatory events. In contrast to the idea of $\mathrm{NBs}$ as separate physical entities from the nuclear genome, our findings highlight the tight interaction between CBs and select regions of the genome and the consequent influence of $\mathrm{CBs}$ on genome organization. $\mathrm{CBs}$ promote a genome conformation that supports co-localization of sn/snoRNA genes distributed throughout the genome, and sustain the highly abundant expression of sn/snoRNA and histone genes. We find that CBs exert a greater regulatory effect on moderately expressed U RNAs, as small U RNAs highly expressed in tissue-matching primary controls were relatively unaffected by CB disassembly. Although the expression of a few spliceosomal small $U$ RNAs has been linked to $\mathrm{CB}$ formation ${ }^{36}$, our study is the first to demonstrate $\mathrm{CB}$-regulated genome-wide effects on the expression profile of sn/snoRNAs and histone genes. In addition to the splicing-related RNA genes, including all major and minor spliceosomal components, all other categories of sn/snoRNA genes are found to be in contact with $\mathrm{CBs}$ and exhibit CB-dependent expression.

In contrast, the global expression profile of protein-coding genes does not significantly depend on CBs. However, several genes related to efficient pre-mRNA splicing show CB-sensitive expression, and may warrant special consideration. Among these was SMC1A whose expression decreased in CB-disrupted cells. In addition to its prominent role in the cohesion complex, which encourages faithful sister chromatid pairing during mitosis, $S M C 1 A$ has also been implicated in the DNA damage response ${ }^{52}$ and may be a potential spliceosome accessory factor ${ }^{33,54}$. SMC1A has also been identified as a mediator of local promoter-enhancer element interactions, as well as chromatin looping ${ }^{55,56}$. Thus, any misregulation of this gene may entail disproportionate effects on the genome architecture and integrity, gene expression, and splicing. Consistent with previous reports on the co-operative relationship between $\mathrm{CBs}$ and spliceosomal core $\mathrm{Sm}$ protein expression, ${ }^{57}$ we also note CB-dependent expression of other genes directly related to the spliceosome, including SNRPE (an integral member of the heptameric Sm-ring complex required for the stability of snRNPs), PRPF3 and SNRNP27 (components of U4/U6.U5 tri-snRNP complex and recycled after each round of RNA splicing), SNRPA1 (U2 snRNP-specific A' protein), and splicing inhibitors such as variant hnRNP H. CBs may also influence DNA and RNA metabolism through the regulation of mRNA synthesis and trafficking (MECP2, MBD1, PEBPC1, THOC5, RPAIN), rRNA and tRNA production (RPL4, POLR1E, EIF6, DUS3L) and DNA damage repair (POLM, SMC1A).

We present evidence for the regulation of splicing fidelity by CBs. Exon and junction analyses of total RNA-seq data show that the noise in the error-prone splicing process increases further when $\mathrm{CBs}$ are disturbed. Consistent with the perturbed expression of spliceosomal components, we observe a substantial usage of alternative exons following CB disassembly, from genes within regions proximal to CBs. In addition, we noted an increased number of aberrant splicing events indicative of errors in splicing. Although junction detection and comparative analysis are computationally challenging, we used relatively deep sequencing, multiple splicing analysis algorithms, and two independent methods of $\mathrm{CB}$ disassembly and found these results suggesting an elevated level of splicing noise in CB-disrupted cells.

Several possible scenarios can be envisioned to link CB function to RNA splicing fidelity. First, loss of CBs and decrease in expression of spliceosomal RNAs result in improper production of spliceosomal complexes. This is unlikely to be the sole contributing factor in this system as spliceosomal snRNPs display long half-lives and is in vast excess ${ }^{58,59}$. Second, improper processing of intron-encoded snoRNAs results in aberrant splice site selection. We found no significant enrichment of intron-encoded $U$ RNAs in those host genes which contain unannotated splicing patterns after $\mathrm{CB}$ disassembly compared with genomic background. Third, CB-dependent rescue of snoRNAs (scaRNAs in particular) from a degradation pathway is lost, resulting in insufficient post-transcriptional base modification of the essential U RNA components of the major and minor spliceosomal pathways. This has previously been linked to changes in splicing fidelity ${ }^{60,61}$ and, in combination with the decreases in U RNA gene expression described in Fig. 1, may explain the changes in splicing we observe following $\mathrm{CB}$ disassembly. Finally, a recent study has indicated that decreases in histone levels, as we report here, may lower co-transcriptional splicing, increase intron retention and induce changes in alternative splicing dynamics ${ }^{48}$. Thus, through HLB and histone gene regulation, CBs may influence RNA maturation.

There may be further downstream genome-wide effects of CBs beyond the phenotypes detected immediately after $\mathrm{CB}$ disassembly in the current study. It is noteworthy that some key regulators of the DNA/RNA metabolism are among the small number of genes with CB-dependent expression (Supplementary Fig. 6). Perhaps a direct regulation of the chromatin landscape may arise through CB's influence over histone gene cluster organization and expression. Interestingly, $\mathrm{CBs}$ also support the expression of SNRPE, an essential component of the Sm ring on U7 snRNP used for histone pre-mRNA processing ${ }^{62}$. Other potential mechanisms that may propagate broadly include poly(A) tail selection, mRNA stability and export, as hinted by altered genes such as THOC5 and PEBPC1. For example, aberrant splicing of THOC5 can have an impact on a large number of genes, since it influences the export and expression of the vast majority of immediate early genes induced by extracellular stimuli. Differential exon usage in the CTD tail of the largest subunit of RNA polymerase II (POLR2A) could also have wide-ranging genomic effects. Taken together, there may be broad longer-term consequences of $\mathrm{CB}$ disassembly beyond the selective changes in gene expression that were observed immediately after CBs are perturbed.

On the basis of the regulation of gene expression and splicing fidelity, we propose a previously unappreciated role of $\mathrm{CBs}$ as 
a

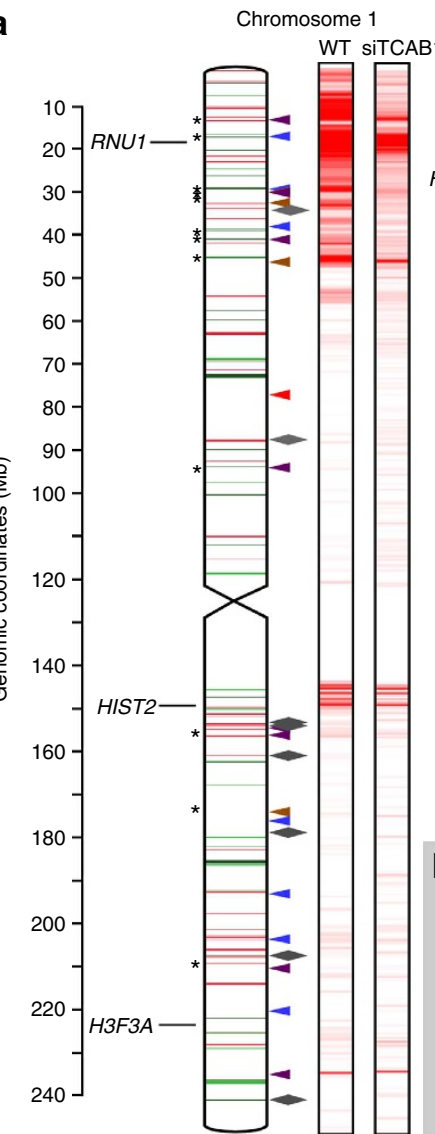

C
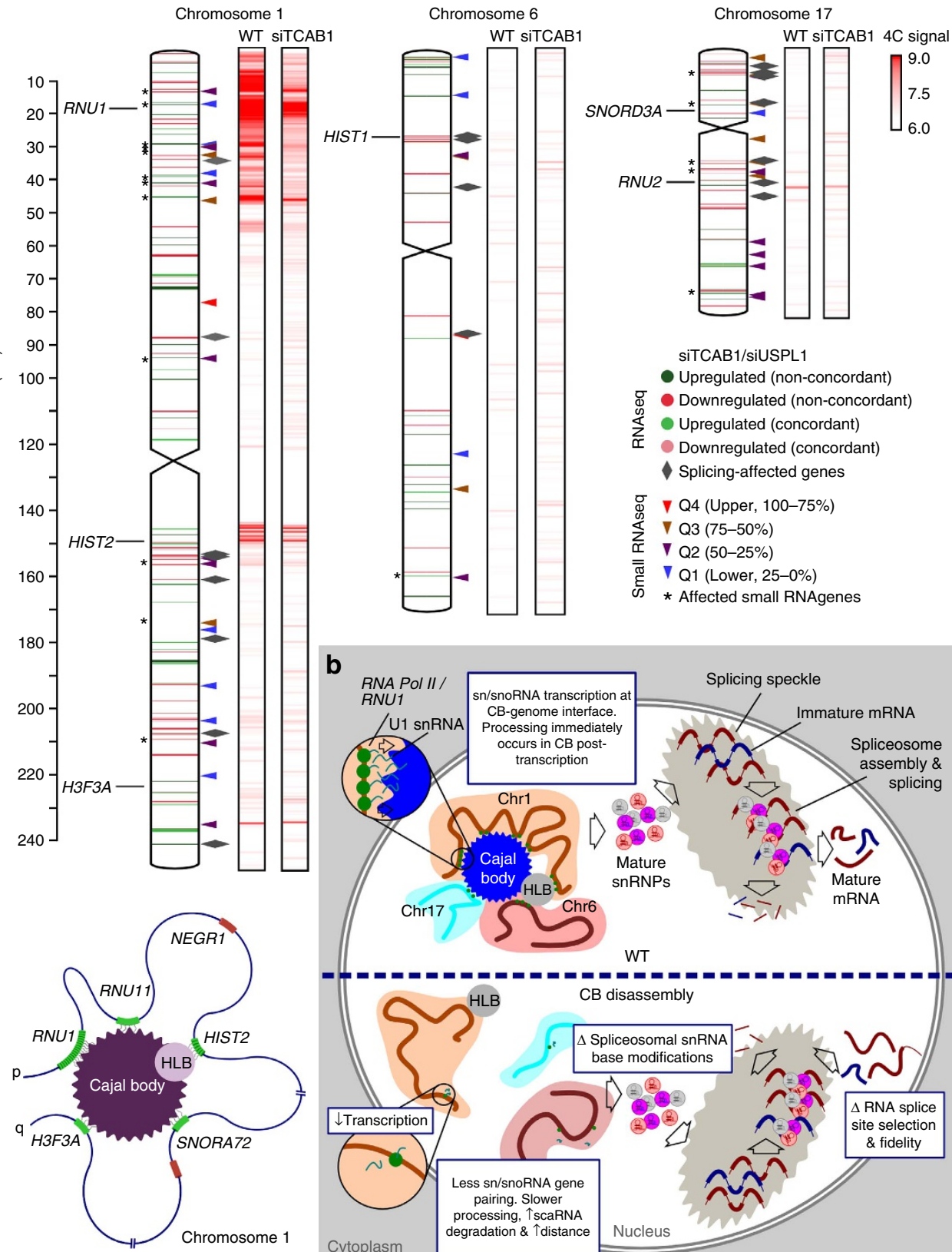

siTCAB1/siUSPL1

- Upregulated (non-concordant)

d Downregulated (non-concordant)

$\sum$ Upregulated (concordant)

○ Downregulated (concordant)

Splicing-affected genes

g V Q4 (Upper, 100-75\%)

I V $3(75-50 \%)$

V Q2 (50-25\%)

气 $\mathrm{\overline { \sigma }}$ Q1 (Lower, 25-0\%)

के * Affected small RNAgenes

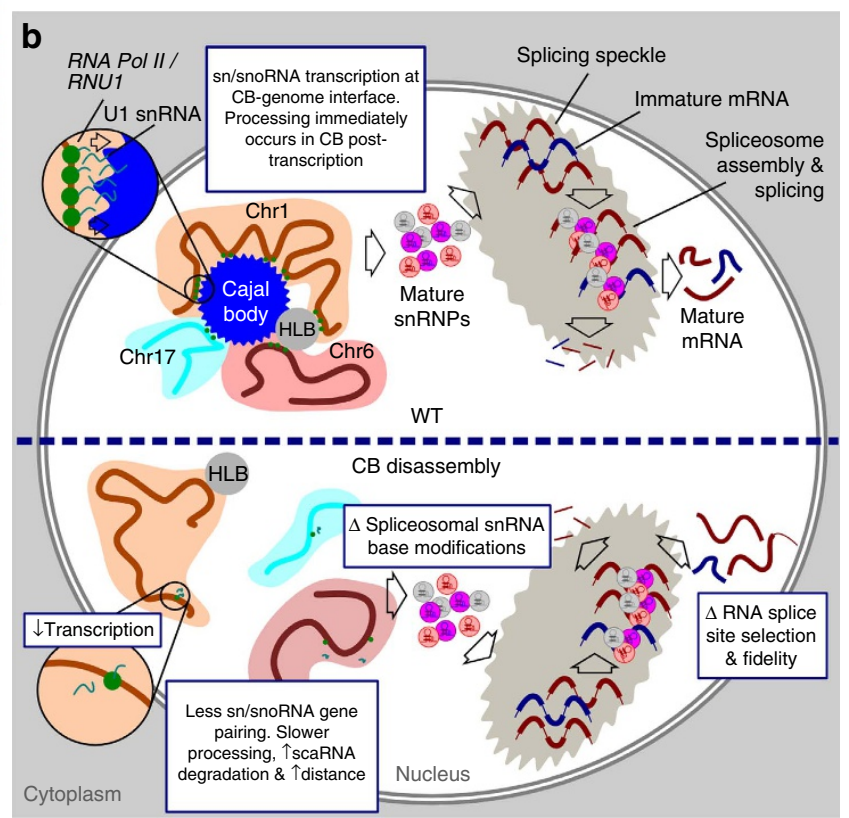

Figure 7 | Cajal bodies orchestrate genome-wide clustering of spliceosomal and histone-related genes. (a) Composite image detailing inter- and intrachromosomal contact $4 \mathrm{C}$ interactions, gene expression changes and alternative splicing events following CB disassembly (siTCAB1) in HeLa cells for chromosomes 1, 6 and 17. The locations of gene loci of interest (RNU1, RNU2, SNORD3A, as well as HIST1, HIST2 and H3F3A) are shown. Upregulated genes (RNA-seq) are denoted by green chromosome bands (non-concordant = dark green, concordant = light green) and downregulated by red (nonconcordant $=$ dark red, concordant $=$ light red). Baseline (siControl) expression of small RNA $U$ genes are denoted by triangles (red $=\mathrm{Q} 4$ (upper, 75-100\%), brown $=\mathrm{Q} 3(50-75 \%)$, purple $=\mathrm{Q} 2(25-50 \%)$ and blue $=\mathrm{Q} 1$ (lower, 0-25\%)) Grey triangles denote the alternative splicing events, ${ }^{*}=$ expression changes of genes (small RNA-seq). 4C-seq physical contact profile is shown in red (4C signal, log10 scale). (b) Proposed role of CBs (blue) in genomic organization and maintenance of tightly regulated splicing processes in HeLa cells. (c) Schematic representation of a proposed topological rearrangement of chromosome 1 by a CB (purple) and physically associated HLB (grey) resulting in regulation of gene positioning. CB- or HLB-interacting gene loci are shown in green, whereas non-interacting regions are shown in red.

genome-organizing centres, in addition to its established function in snRNP biogenesis and recycling pathways. Our data also establish that highly expressed, frequently $\mathrm{CB}$-proximal small $\mathrm{U}$ RNA gene loci, such as the highly expressed RNU1 or RNU2 gene arrays (Fig. 7a), probably serve as key $\mathrm{CB}$ nucleation sites capable of targeting all the other categories of small RNA genes. Once structurally formed, CBs promote the looping and aggregation of a limited cohort of sn/snoRNA and histone (via the physically associated HLBs) genes in their proximity, particularly within chromosome 1 , to regulate their expression (Fig. $7 b, c)^{37}$. In the absence of $\mathrm{CBs}$, these topological repositioning events do not occur, resulting in downregulation of small U RNAs and changes in genome-wide chromosomal interactions. Depletion of spliceosomal U RNA genes and scaRNAs that guide their base modifications is likely to alter RNA splicing fidelity, as previously described $^{63}$. Our study opens the way for further investigations of 
the longitudinal effects of $\mathrm{CB}$ formation and disruption in a controlled system.

\section{Methods}

Cell culture. HeLa human cervical carcinoma cells were maintained according to standard procedures in DMEM containing $10 \%(\mathrm{v} / \mathrm{v})$ fetal bovine serum, glucose $\left(4.5 \mathrm{gl}^{-1}\right)$, and $5 \% \mathrm{CO}_{2}$. The primary human cervical epithelial cell line (Epi) was purchased from Cell Applications and maintained in propriety Epi growth medium $/ 5 \% \mathrm{CO}_{2}$. D551 primary skin fibroblasts were kindly provided by Dr Dominik Duelli and cultured under the same conditions as HeLa cells. One day before lysis, Epi cells were transferred to DMEM and maintained under the same conditions as HeLa and D551 cells to account for differences arising from culture conditions.

\section{siRNA oligonucleotide transfection and cell cycle arrest. Delivery of siRNA} into HeLa cells was achieved using DharmaFECT 1 transfection reagent (Dharmacon) according to manufacturer's instructions. TCAB1 siRNA was acquired from Qiagen (SI00388941), USPL1 siRNA (sc-76875) and non-targeting control siRNA (sc-37007) from Santa Cruz. Briefly, $100 \mathrm{nM}$ siRNA was transfected on two consecutive days and cells were collected $24 \mathrm{~h}$ after the second round of transfection.

To induce an artificial cell cycle block at the G1/S-phase boundary, HeLa cells were treated with a final concentration of $5 \mathrm{mM}$ thymidine for $18 \mathrm{~h}$. Cells were released and grown in fresh media for $9 \mathrm{~h}$, followed by a second block with $5 \mathrm{mM}$ thymidine for $18 \mathrm{~h}$. Cells were immediately collected for analysis after the second thymidine block without release into fresh media.

Hybridization probes. Probes were purchased from BACPAC, tested by PCR, metaphase spreads and co-staining with CTs in primary diploid cells. The DNA hybridization probes were generated using specific BACs by nick translation with 488-dUTP from Abbott Laboratories, 552-dUTP and Cy5-dUTP from GE Healthcare, and 594-dUTP and 680-dUTP from Biotium.

DNA FISH. Cells grown on glass $12 \mathrm{~mm}$ circle coverslips were washed with PBS and fixed with $4 \%$ PFA in PBS for $10 \mathrm{~min}$ at room temperature. After rinsing in PBS, cells were permeabilized with $0.5 \%$ Triton X-100 in PBS for $20 \mathrm{~min}$ on ice and were washed with PBS. Subsequently, the cells were incubated in $0.1 \mathrm{~N} \mathrm{HCl}$ for $15 \mathrm{~min}$, and then washed twice in $2 \times$ SSC for $10 \mathrm{~min}$, before equilibration in $50 \%$ formamide $/ 2 \times \mathrm{SSC}$ for $30 \mathrm{~min}$. For one $12-\mathrm{mm}$ round coverslip, $\sim 150 \mathrm{ng}$ of DNA FISH probe of each fluorescent dye were combined together and precipitated with $3 \mu \mathrm{g}$ of Cot-1 DNA (Roche) and $1 \mu \mathrm{g}$ of yeast tRNA in ice-cold absolute ethanol by sodium acetate, spun down, dried and resuspended in $7 \mu$ lof hybridization solution (10\% dextran sulfate/50\% formamide $(\mathrm{pH} 7.0) / 2 \times \mathrm{SSC} / 1 \%$ Tween 20$)$. The cocktail was denatured at $85^{\circ} \mathrm{C}$ for $5 \mathrm{~min}$, put briefly on ice, incubated with cells at $85^{\circ} \mathrm{C}$ for $5 \mathrm{~min}$, sealed with rubber cement and hybridized in a humidified chamber overnight at $37^{\circ} \mathrm{C}$. After hybridization, cells were washed three times in $50 \%$ formamide $/ 2 \times \mathrm{SSC}$ for $5 \mathrm{~min}$ at $45^{\circ} \mathrm{C}$, washed three times in $1 \times \mathrm{SSC}$ for $5 \mathrm{~min}$ at $60^{\circ} \mathrm{C}$ and in PBS for $5 \mathrm{~min}$. Then cells were incubated with the rabbit antibody against coilin (Santa Cruz) for $1 \mathrm{~h}$, washed three times in PBS. Cells were then incubated with donkey anti-rabbit secondary antibody conjugated with $405 \mathrm{~S}$ dye (Jackson ImmunoResearch Laboratories) for $1 \mathrm{~h}$, washed three times in PBS for $5 \mathrm{~min}$, and mounted using ProLong antifade mounting reagent without DAPI (Life Technologies). Cells were observed on Zeiss LSM710 or LSM780 confocal microscope using a $63 \times 1.40 \mathrm{NA}$ objective using $\mathrm{z}$-sectioning $(\mathrm{z}-250 \mathrm{~nm})$ with spectral imaging and linear unmixing using Zeiss Zen software.

3D DNA chromosome painting. The protocol is identical to DNA FISH procedure except chromosome painting probes were hybridized with fixed cells for three nights at $37^{\circ} \mathrm{C}$. Theoretical association frequencies were assessed after quantification of the nuclear and CT1 areas using ImageJ. The radii of these areas were then estimated and used with the following formula:

$$
\text { Expected association frequency }(\%)=100 \times \frac{(\mathrm{CT} 1 \mathrm{rad}+\Delta)^{2}}{(\mathrm{NUCrad})^{2}}
$$

CT1 $\mathrm{rad}=$ average radius of chromosome $1, \mathrm{NUCrad}=$ average nuclear radius $\Delta=$ threshold for edge-edge distance between CB and Chrl

Chromosome conformation capture followed by sequencing. The $4 \mathrm{C}$ assay was performed as previously described ${ }^{64}$. Briefly, cultured HeLa cells were cross-linked in $2 \%$ formaldehyde at $37^{\circ} \mathrm{C}$ for $10 \mathrm{~min}$. After being quenched with glycine, cells were lysed using $10 \mathrm{mM}$ Tris- $\mathrm{HCl}, \mathrm{pH} 8.0,10 \mathrm{mM} \mathrm{NaCl}, 0.2 \% \mathrm{NP}-40$ ) supplemented with $1 \times$ complete protease inhibitors (Sigma) at $4{ }^{\circ} \mathrm{C}$ for $1 \mathrm{~h}$. Cross-linked chromatin was digested overnight with an excess of HindIII enzyme (New England Biolabs), and then DNA ends were ligated under diluted conditions that favour junctions between cross-linked DNA fragments. NlaIII (New England Biolabs) was used in a second round of digestion, and DNA was re-ligated. Primers were used to PCR-amplify 4C DNA can be found in Supplementary Data 1.
Each experiment was independently performed twice. 4C DNA samples were barcoded and sequenced on Illumina HiSeq2000 according to the Illumina paired-end $100 \mathrm{bp}$ sequencing protocol.

$4 \mathrm{C}$-seq data were analysed by the following procedure: Raw data were initially processed by de-multiplexing, bait-trimming and quality filtering. Reads were trimmed to the first 50 nucleotides before mapping them onto hg19 using Bowtie. A primary $4 \mathrm{C}$ profile was generated by counting the number of reads overlapping each non-overlapping genomic bin of size $10 \mathrm{~kb}$. For smoothed $4 \mathrm{C}$ profiles, the running $100 \mathrm{~kb}$ window-average of the bin counts was assigned to the centre of each genomic $10 \mathrm{~kb}$ bin. After confirming profile reproducibility, duplicate $4 \mathrm{C}$ files were pooled by combining reads from two biological replicates for each condition. The smoothed $4 \mathrm{C}$ profiles were generated from the pooled data, and 0.1 was added before converting to the $\log 2$ scale.

Enrichment of small RNA and histone genes within $10 \mathrm{~kb}$ of $4 \mathrm{C}$ contacts was assessed by generating 100 random samples of matching size and calculating an empirical $P$ value. For $4 \mathrm{C}$ contact regions, we retrieved the coordinates of the smoothed profile with the above-defined $\log 24 \mathrm{C}$ signal $>7$.

RNA preparation and qRT-PCR. Total cellular RNA was extracted with RNeasy Mini kit (Qiagen) in accordance with the manufacturer's instructions, before sequencing. For quantitative RT-PCR, cDNA was synthesized using the iScript cDNA Synthesis kit (Bio-Rad). Real-time PCR was performed in triplicate using the iQ SYBR Green PCR Supermix (Bio-Rad). The abundances of the mRNAs of interest in each sample were normalized to that of $\beta$-actin mRNA, and fold changes in target mRNAs relative to their basal abundances were calculated by the $2^{-\Delta \Delta \mathrm{Ct}}$ method. Each experiment was performed in two independent repeats.

Small RNA sample preparation and sequencing. Ribosomal RNAs (rRNA) were removed from total RNA samples using Ribo-Zero rRNA Removal Kits (Epicentre). The total RNA samples (after depletion of rRNA) were size-fractionated on a $6 \%$ TBE-Urea polyacrylamide gel to enrich for molecules in the range of 50-400 nt. Small RNA libraries and sequencing for each sample were performed by LC Sciences (Houston, TX). In brief, RNA fragments were dephosphorylated and ligated with $3^{\prime}$-RNA adaptor. The resulting ligated RNA fragments were reversed-transcribed and ligated with $5^{\prime}$-DNA/DNA adaptor and PCR amplified (15 cycles) using primers on both adaptors. Subsequently, PCR products were purified and small RNA library was sequenced on Illumina GAIIx (Illumina, Santa Clara, CA) for single-end 35-mers or on Illumina HiSeq for single-end 51-mers following the manufacturer's protocol. Raw sequence FASTQ files were generated by using Illumina's software pipeline.

Small RNA-seq data analysis. Raw reads were adaptor-trimmed and filtered to remove low-quality reads. We downloaded the hg19 coordinates for all the annotated snRNAs from the UCSC browser based on ENSEMBLE version 70 (January 2013) and a query for RNU, snoRNA, snoRD and scaRNA. This resulted in 1,649 non-redundant small RNAs and their coordinates. In all, 35-mers were mapped to the hg19 reference genome using Bowtie 1.0.0 with the parameters '-p 4 -v 1 -m 1'. We computed fragments per kilobase per million mapped reads (FPKM) using Cufflinks over the annotated small RNAs using the downloaded coordinates. We also verified that the numbers of reads mapping to mRNAs were negligible as expected from the size selection. After confirming reproducibility of global profiles in biological replicates, the average of the duplicate log2 FPKM values was used to quantify small RNA expression. Expression in a knock-down sample was compared with expression in untreated HeLa (control 1) and in siControl-treated HeLa (control 2). An increase/decrease in expression was deemed significant if it is relative to both control 1 and control 2 .

RNA-seq sequencing and genome alignment. Libraries were generated with the Illumina Truseq protocol (Illumina, San Diego, CA) and TruSeq sample prep kits (catalogue ID: FC-122-1001). Libraries were sequenced on a HiSeq2000 instrument (Illumina) for $2 \times 101$ cycles, generating 101 base-paired-end reads. All samples were run on the same flowcell, multiplexed with 4 samples per lane. The Illumina basecalling and read generation algorithm versions were RTA 1.12.4.2 and CASAVA-1.8.2. Sequencing, read pre-processing and alignment were performed by the Center for Cancer Research (CCR) Genomics Core (Frederick, MD). Raw reads were trimmed before alignment. The trimming software used was ea-utils FasqMcf software (http://code.google.com/p/ea-utils/wiki FastqMcf Expression Analysis, Durham, NC). The trimming parameters used were: -l 15 -q 0 -u -P 33 (minimum retained sequence length $=15$, quality threshold causing base removal $=0$, enable Illumina PF filtering, Phred 33 scale). Trimmed reads were mapped to the hg19 reference genome with TopHat v.2.0.8 (ref. 65, accepting only unique alignments (parameters -g 1 -r 10 --mate-std-dev 100), with the Bowtie2 alignment engine ${ }^{66}$. Gene model annotation for hg19 from Ensembl was provided to the aligner with the $-\mathrm{G}$ parameter.

List of contact genes from 4C data. For each 4C experiment (RNU1 and H3F3A), lists of putative interacting genes were obtained as follows: from single-base positions, BED files of extended ranges $( \pm 10 \mathrm{~kb}, 25 \mathrm{~kb}$ and $50 \mathrm{~kb})$ were generated. A 
BED file of extended gene regions (start to end) was generated from UCSC annotation and the R package 'rtracklayer' and 'GenomicFeatures'. We performed intersections of these BED files with bedTools ${ }^{67}$, requiring that the midpoint of the extended gene overlap with the $4 \mathrm{C}$ region (intersectBed $-\mathrm{f} 0.50$ ). From overlapping genes, we applied a gene expression criterion to define putative 4C-positive interactors (gene expression in wild type in the top $5 \%$ of all genes). A control set (4C-negative) was defined as genes in the top 5\% of gene expression in wild type, but residing $>50 \mathrm{~kb}$ from $4 \mathrm{C}$ contact regions. Lists are included in Supplementary Data 1.

RNA-seq data analysis. All expression analysis was performed against the UCSC hg19 genes.gtf annotation, downloaded from the iGenomes collection (Illumina). Gene-level expression in units of FPKM were generated with Cufflinks v.2.1.1 (ref. 68, using default parameters except for turning off suppression of rare isoforms (-F 0). Differential expression was performed using Cuffdiff v.2.1.1, using 'quartile' as the library normalization method. Gene expression was also quantified using the HTSeq program v.0.5.4p1 (htseq-count parameters $-\mathrm{q}-\mathrm{m}$ union $-\mathrm{s}$ no $-\mathrm{i}$ gene_id -t exon). Count results were analysed with DEseq v.1.16 (ref. 45). We used these results to generate a high-confidence set of gene expression calls based on concordance between the two methods (Supplementary Data 3). Complete genelevel output from each program is provided in Supplementary Data 3.

Splicing was analysed by multiple complementary approaches. We used the Splicing Analysis Toolkit (Spanki) v.0.4.3 (ref. 69) to quantify splice junction coverage from alignment files, and to quantify proportion spliced in for splicing events. The AStalavista tool ${ }^{70}$ was used to characterize events from annotated transcript models. This tool requires annotation in a GTF format only available for annotation distributed by Ensembl. We downloaded the most recent Ensembl annotation (Homo_sapiens.GRCh37.75.gtf), and identified 638,467 pairwise splicing events with AStalavista v.3.2. The Spanki toolkit was also used to assign unannotated splice junctions to genes. Splicing was also quantified using the Jensen-Shannon Divergence metric from the Cuffdiff program ${ }^{68}$. To analyse unannotated splice junctions unique to knockdown, we generated two metrics to normalize junction detection based on transcription and sequencing depth.

Count-based analysis within exons was performed with the DEXSeq package ${ }^{71}$, which quantifies exon expression normalized for transcription and sequencing depth $\left(\mathrm{KD} 1 \_\right.$reads_per_million $=$coverage in $\mathrm{KD} 1 /$ millions of reads,

Wt_Expect_KD1_depth $=$ millions of reads ${ }^{*} \mathrm{KD} 1$ reads_per million, KD1_reads_per_FPKM $=$ coverage in KD1/FPKM in KD1,

Wt_Expect_KD1_FPKM $=$ FPKM ${ }^{*}$ KD1_reads_per_FPKM). Gene ontology functional enrichment analysis was performed with the DAVID tool ${ }^{72}$ and the GOStats tool ${ }^{73}$.

High-throughput microscopy. Following DNA FISH/IF staining as described above, except for seeding of cells in 384-well clear-bottom plates (CellCarrier, PerkinElmer), cells were imaged using an Opera QEHS (PerkinElmer) spinning-disk confocal high-throughput imaging system. Images were acquired in three sequential exposures in four channels:

(i) DAPI: excitation laser $405 \mathrm{~nm}$, emission filter: BP 450/50, exposure 1.

(ii) RNU1-488 nm: excitation laser $488 \mathrm{~nm}$, emission filter: BP 520/35, exposure 3.

(iii) Variable gene of interest (VGOI) BAC-568nm: excitation laser $561 \mathrm{~nm}$, emission filter: BP $600 / 40$, exposure 2.

(iv) Cy5: excitation laser $640 \mathrm{~nm}$, emission filter: BP 600/40, exposure 1. All channels were acquired using a 405/488/561/640 primary excitation dichroic mirror and a 568 detection dichroic mirror. The images were acquired using a $\times 40$ water objective lens (NA 0.9 , focal depth $1.2 \mu \mathrm{m}$ ) with 6 optical steps of $1.0 \mu \mathrm{m}$, with a camera binning of 2 . The pixel size was $320 \mathrm{~nm}$.

Automated image analysis. Images were analysed with the Acapella 2.0 or 2.6 software (PerkinElmer). To quantify distances between FISH signals and coilin, we modified a custom image analysis script in Acapella for nuclear segmentation and spot detection ${ }^{74}$. In brief, images from different $z$-planes and from the same channel and field of view were maximally projected in 2D. Nuclei were segmented using the maximally projected images in the DAPI channel. Nuclei touching the image border were not considered for further analysis. The segmented nuclear region was then used by an intensity-based spot detection algorithm to segment the FISH and IF signals in the maximally projected RNU1-488 nm and VGOI-568nm channels and the Coilin bodies were detected using the maximally projected $\mathrm{Cy} 5$ channel. For every FISH and IF signal, its brightest pixel was identified as the spot centre. All the distances between spots of different colours were measured by calculating the Euclidean distance between their spot centres using their respective $x$ - and $y$-axis pixel coordinates in the image. All the single-cell and single-distance data was indexed on a per-well, per-imaging field, per-cell and per-spot basis for further downstream analysis. Single-object data (Cell- and distance-level) was exported as '.txt files'. The Acapella 2.0 or 2.6 image analysis scripts and analysis parameters, as well as all microscopy images generated during this project, are available on request to the authors.
Single-distance data analysis. Data analysis on single-object level data was performed using the $\mathrm{R}$ statistical analysis software ${ }^{75}$. In brief, only spot distances from cells with at least 1 FISH signal per gene and 1 CB were considered for further analysis. Minimum RNU1-gene of interest and RNU1-CB were calculated on a per RNU1 FISH spot basis. CB-gene of interest distances were calculated on a per-CB spot basis. Since all the distances were indexed based on their identity of the spots they were calculated from, it was possible to assign a minimum CB-gene of interest basis distance for a particular RNU1 FISH Spot based on its minimum RNU1 FISH Spot/CB minimum distance. A CB-independent RNU1-gene of interest interaction was defined as a RNU1 FISH Spot having a minimum RNU1/gene of interest distance of $\leq 0.96 \mu \mathrm{m}$ ( 3 pixels), and a minimum RNU1/CB distance of $>1.6 \mu \mathrm{m}$ (5 pixels, threshold distances were selected following visual scoring of images before automated analysis, Supplementary Fig. 4d,e). A CB-dependent RNU1-Gene of Interest interaction was defined as a RNU1 FISH Spot having a minimum RNU1/gene of interest distance of $\leq 0.96 \mu \mathrm{m}$ (3 pixels), and a minimum RNU1/CB distance of $\leq 1.6 \mu \mathrm{m}$ (5 pixels). A CB bridging event was defined as a RNU1 FISH Spot having a minimum RNU1/CB distance of $\leq 1.6 \mu \mathrm{m}$ (5 pixels), and the respective minimum $\mathrm{CB} / \mathrm{VGOI}$ distance of $\leq 1.6 \mu \mathrm{m}$ (5 pixels). Interaction frequencies between each of the genes and RNU1 were calculated by computing the percentage of cells carrying at least one event as defined by the criteria listed above. The '.rmd' file containing the R script using for the single-distance data analysis is available on request.

\section{References}

1. Fraser, P. \& Bickmore, W. Nuclear organization of the genome and the potential for gene regulation. Nature 447, 413-417 (2007).

2. Cremer, T. \& Cremer, M. Chromosome territories. Cold Spring Harb. Perspect. Biol. 2, a003889 (2010)

3. Lieberman-Aiden, E. et al. Comprehensive mapping of long-range interactions reveals folding principles of the human genome. Science 326, 289-293 (2009).

4. Cavalli, G. \& Misteli, T. Functional implications of genome topology. Nat. Struct. Mol. Biol. 20, 290-299 (2013).

5. Dundr, M. \& Misteli, T. Biogenesis of nuclear bodies. Cold Spring Harb. Perspect. Biol. 2, a000711 (2010).

6. Spector, D. L. \& Lamond, A. I. Nuclear speckles. Cold Spring Harb. Perspect. Biol. 3, a000646 (2011).

7. Machyna, M., Heyn, P. \& Neugebauer, K. M. Cajal bodies: where form meets function. Wiley Interdiscip. Rev. RNA 4, 17-34 (2013).

8. Sleeman, J. E. \& Trinkle-Mulcahy, L. Nuclear bodies: new insights into assembly/dynamics and disease relevance. Curr. Opin. Cell Biol. 28, 76-83 (2014).

9. Mao, Y. S., Zhang, B. \& Spector, D. L. Biogenesis and function of nuclear bodies. Trends Genet. 27, 295-306 (2011).

10. Frey, M. R. \& Matera, A. G. RNA-mediated interaction of Cajal bodies and U2 snRNA genes. J. Cell Biol. 154, 499-509 (2001).

11. Mao, Y. S., Sunwoo, H., Zhang, B. \& Spector, D. L. Direct visualization of the co-transcriptional assembly of a nuclear body by noncoding RNAs. Nat. Cell Biol. 13, 95-101 (2011)

12. Shevtsov, S. P. \& Dundr, M. Nucleation of nuclear bodies by RNA. Nat. Cell Biol. 13, 167-173 (2011).

13. Grob, A., Colleran, C. \& McStay, B. Construction of synthetic nucleoli in human cells reveals how a major functional nuclear domain is formed and propagated through cell division. Genes Dev. 28, 220-230 (2014).

14. Tan, C., Saurabh, S., Bruchez, M. P., Schwartz, R. \& Leduc, P. Molecular crowding shapes gene expression in synthetic cellular nanosystems. Nat. Nanotechnol. 8, 602-608 (2013).

15. Cho, E. J. \& Kim, J. S. Crowding effects on the formation and maintenance of nuclear bodies: insights from molecular-dynamics simulations of simple spherical model particles. Biophys. J. 103, 424-433 (2012).

16. ten Wolde, P. R. \& Mugler, A. in International Review of Cell and Molecular Biology Volume 307 (eds Ronald, H. \& Kwang, W. J.) 419-442 (Academic Press, 2014).

17. Matera, A. G., Izaguire-Sierra, M., Praveen, K. \& Rajendra, T. K. Nuclear bodies: random aggregates of sticky proteins or crucibles of macromolecular assembly? Dev. Cell 17, 639-647 (2009).

18. Dundr, M. Nuclear bodies: multifunctional companions of the genome. Curr Opin. Cell Biol. 24, 415-422 (2012).

19. Matera, A. G. \& Wang, Z. A day in the life of the spliceosome. Nat. Rev. Mol. Cell Biol. 15, 108-121 (2014).

20. Klingauf, M., Stanek, D. \& Neugebauer, K. M. Enhancement of U4/U6 small nuclear ribonucleoprotein particle association in Cajal bodies predicted by mathematical modeling. Mol. Biol. Cell 17, 4972-4981 (2006).

21. Novotny, I., Blazikova, M., Stanek, D., Herman, P. \& Malinsky, J. In vivo kinetics of U4/U6.U5 tri-snRNP formation in Cajal bodies. Mol. Biol. Cell 22, 513-523 (2011)

22. Smith, K. P., Carter, K. C., Johnson, C. V. \& Lawrence, J. B. U2 and U1 snRNA gene loci associate with coiled bodies. J. Cell. Biochem. 59, 473-485 (1995). 
23. Frey, M. R. \& Matera, A. G. Coiled bodies contain U7 small nuclear RNA and associate with specific DNA sequences in interphase human cells. Proc. Natl Acad. Sci. USA 92, 5915-5919 (1995).

24. Gao, L., Frey, M. R. \& Matera, A. G. Human genes encoding U3 snRNA associate with coiled bodies in interphase cells and are clustered on chromosome 17p11.2 in a complex inverted repeat structure. Nucleic Acids Res. 25, 4740-4747 (1997).

25. Jacobs, E. Y. et al. Coiled bodies preferentially associate with U4, U11, and U12 small nuclear RNA genes in interphase HeLa Cells but Not with U6 and U7 Genes. Mol. Biol. Cell 10, 1653-1663 (1999).

26. James Faresse, N. et al. Genomic study of RNA polymerase II and III SNAPcbound promoters reveals a gene transcribed by both enzymes and a broad use of common activators. PLoS Genet 8, e1003028 (2012).

27. Dieci, G., Preti, M. \& Montanini, B. Eukaryotic snoRNAs: a paradigm for gene expression flexibility. Genomics 94, 83-88 (2009).

28. Shopland, L. S. et al. Replication-dependent histone gene expression is related to cajal body (CB) association but does not require sustained CB contact. Mol. Biol.Cell 12, 565-576 (2001).

29. Nizami, Z., Deryusheva, S. \& Gall, J. G. The Cajal body and histone locus body. Cold Spring Harb. Perspect. Biol. 2, a000653 (2010).

30. Salzler, H. R. et al. A sequence in the Drosophila H3-H4 promoter triggers histone locus body assembly and biosynthesis of replication-coupled histone mRNAs. Dev. Cell 24, 623-634 (2013).

31. O'Reilly, D. et al. Differentially expressed, variant U1 snRNAs regulate gene expression in human cells. Genome Res. 23, 281-291 (2013).

32. DeRan, M., Pulvino, M., Greene, E., Su, C. \& Zhao, J. Transcriptional activation of histone genes requires NPAT-dependent recruitment of TRRAP-Tip60 complex to histone promoters during the G1/S phase transition. Mol. Cell Biol. 28, 435-447 (2008).

33. Venteicher, A. S. et al. A human telomerase holoenzyme protein required for Cajal body localization and telomere synthesis. Science 323, 644-648 (2009).

34. Mahmoudi, S. et al. WRAP53 is essential for Cajal body formation and for targeting the survival of motor neuron complex to Cajal bodies. PLoS Biol. 8, e1000521 (2010).

35. Schulz, S. et al. Ubiquitin-specific protease-like 1 (USPL1) is a SUMO isopeptidase with essential, non-catalytic functions. EMBO Rep. 13, 930-938 (2012).

36. Hutten, S., Chachami, G., Winter, U., Melchior, F. \& Lamond, A. I. A role for the Cajal-body-associated SUMO isopeptidase USPL1 in snRNA transcription mediated by RNA polymerase II. J. Cell Sci. 127, 1065-1078 (2014).

37. Dundr, M. et al. Actin-dependent intranuclear repositioning of an active gene locus in vivo. J. Cell Biol. 179, 1095-1103 (2007).

38. Simonis, M. et al. Nuclear organization of active and inactive chromatin domains uncovered by chromosome conformation capture-on-chip (4C). Nat. Genet. 38, 1348-1354 (2006).

39. Teves, S. S., Weber, C. M. \& Henikoff, S. Transcribing through the nucleosome. Trends Biochem. Sci. 39, 577-586 (2014).

40. Tessereau, C. et al. Direct visualization of the highly polymorphic RNU2 locus in proximity to the BRCA1 gene. PLoS One 8, e76054 (2013).

41. Williamson, I. et al. Spatial genome organization: contrasting views from chromosome conformation capture and fluorescence in situ hybridization. Genes Dev. 28, 2778-2791 (2014).

42. Yuan, P. et al. Telomerase Cajal body protein 1 depletion inhibits telomerase trafficking to telomeres and induces G cell cycle arrest in A549 cells. Oncol. Lett. 8, 1009-1016 (2014).

43. Chen, J. \& Wagner, E. J. snRNA 3' end formation: the dawn of the Integrator complex. Biochem. Soc. Trans. 38, 1082-1087 (2010).

44. Takata, H., Nishijima, H., Maeshima, K. \& Shibahara, K. The integrator complex is required for integrity of Cajal bodies. J. Cell Sci. 125, 166-175 (2012).

45. Anders, S. \& Huber, W. Differential expression analysis for sequence count data. Genome Biol. 11, R106 (2010).

46. Bass, B. L. RNA editing by adenosine deaminases that act on RNA. Annu. Rev. Biochem. 71, 817-846 (2002).

47. Meinhart, A. \& Cramer, P. Recognition of RNA polymerase II carboxy-terminal domain by 3'-RNA-processing factors. Nature 430, 223-226 (2004).

48. Jimeno-Gonzalez, S. et al. Defective histone supply causes changes in RNA polymerase II elongation rate and cotranscriptional pre-mRNA splicing. Proc. Natl Acad. Sci. USA 112, 14840-14845 (2015).

49. Kaiser, T. E., Intine, R. V. \& Dundr, M. De novo formation of a subnuclear body. Science 322, 1713-1717 (2008).

50. Platani, M., Goldberg, I., Lamond, A. I. \& Swedlow, J. R. Cajal body dynamics and association with chromatin are ATP-dependent. Nat. Cell Biol. 4, 502-508 (2002).
51. Machyna, M. et al. The coilin interactome identifies hundreds of small noncoding RNAs that traffic through Cajal bodies. Mol. Cell 56, 389-399 (2014).

52. Watrin, E. \& Peters, J. M. The cohesin complex is required for the DNA damage-induced G2/M checkpoint in mammalian cells. EMBO J. 28, 2625-2635 (2009).

53. Hartmuth, K. et al. Protein composition of human prespliceosomes isolated by a tobramycin affinity-selection method. Proc. Natl Acad. Sci. USA 99, 16719-16724 (2002).

54. Sharma, S., Kohlstaedt, L. A., Damianov, A., Rio, D. C. \& Black, D. L. Polypyrimidine tract binding protein controls the transition from exon definition to an intron defined spliceosome. Nat. Struct. Mol. Biol. 15, 183-191 (2008).

55. DeMare, L. E. et al. The genomic landscape of cohesin-associated chromatin interactions. Genome Res. 23, 1224-1234 (2013).

56. Kagey, M. H. et al. Mediator and cohesin connect gene expression and chromatin architecture. Nature 467, 430-435 (2010).

57. Lemm, I. et al. Ongoing U snRNP biogenesis is required for the integrity of Cajal bodies. Mol. Biol. Cell 17, 3221-3231 (2006).

58. Lund, E. \& Dahlberg, J. E. True genes for human U1 small nuclear RNA. Copy number, polymorphism, and methylation. J. Biol. Chem. 259, 2013-2021 (1984).

59. Tardiff, D. F. \& Rosbash, M. Arrested yeast splicing complexes indicate stepwise snRNP recruitment during in vivo spliceosome assembly. RNA 12, 968-979 (2006).

60. Karijolich, J. \& Yu, Y. T. Spliceosomal snRNA modifications and their function RNA Biol. 7, 192-204 (2010).

61. Yu, Y. T. \& Meier, U. T. RNA-guided isomerization of uridine to pseudouridine--pseudouridylation. RNA Biol. 11, 1483-1494 (2014).

62. Tisdale, S. et al. SMN is essential for the biogenesis of U7 small nuclear ribonucleoprotein and 3'-end formation of histone mRNAs. Cell Rep. 5, 1187-1195 (2013).

63. Wu, G., Yu, A. T., Kantartzis, A. \& Yu, Y. T. Functions and mechanisms of spliceosomal small nuclear RNA pseudouridylation. Wiley Interdiscip. Rev. RNA 2, 571-581 (2011).

64. Hakim, O. et al. Diverse gene reprogramming events occur in the same spatial clusters of distal regulatory elements. Genome Res. 21, 697-706 (2011).

65. Trapnell, C., Pachter, L. \& Salzberg, S. L. TopHat: discovering splice junctions with RNA-Seq. Bioinformatics 25, 1105-1111 (2009).

66. Langmead, B. \& Salzberg, S. L. Fast gapped-read alignment with Bowtie 2. Nat Methods 9, 357-359 (2012)

67. Quinlan, A. R. \& Hall, I. M. BEDTools: a flexible suite of utilities for comparing genomic features. Bioinformatics 26, 841-842 (2010).

68. Trapnell, C. et al. Transcript assembly and quantification by RNA-Seq reveals unannotated transcripts and isoform switching during cell differentiation. Nat. Biotechnol. 28, 511-515 (2010).

69. Sturgill, D. et al. Design of RNA splicing analysis null models for post hoc filtering of Drosophila head RNA-Seq data with the splicing analysis kit (Spanki). BMC Bioinformatics 14, 320 (2013).

70. Sammeth, M., Foissac, S. \& Guigo, R. A general definition and nomenclature for alternative splicing events. PLoS Comput. Biol. 4, e1000147 (2008).

71. Anders, S., Reyes, A. \& Huber, W. Detecting differential usage of exons from RNA-seq data. Genome Res. 22, 2008-2017 (2012).

72. Huang, D. W., Sherman, B. T. \& Lempicki, R. A. Systematic and integrative analysis of large gene lists using DAVID Bioinformatics Resources. Nature Protoc. 4, 44-57 (2009).

73. Beißbarth, T. \& Speed, T. P. GOstat: find statistically overrepresented gene ontologies within a group of genes. Bioinformatics 20, 1464-1465 (2004).

74. Roukos, V. et al. Spatial dynamics of chromosome translocations in living cells. Science 341, 660-664 (2013).

75. R: A language and environment for statistical programming v. 3.1.2 (R Foundation for Statistical Computing, 2014).

\section{Acknowledgements}

We are particularly grateful to T. Misteli for helpful discussions. We thank T. Misteli and S. Oberdoerffer for critical reading of the manuscript. Analysis of high throughput sequencing data was partly performed on the NIH supercomputing cluster Biowulf. This research is supported by the Intramural Research Program of NIH (M.-H.S., S.B., S.P.S., G.P., G.L.H.) and NIH R01GM090156 from NIGMS (M.D.)

\section{Author contributions}

Q.W., M.-H.S., D.S., G.L.H. and M.D. designed the project. S.P.S. and M.D. performed DNA FISH microscopy and quantitative analysis. Q.W. and O.H. conducted 4C-seq, and small- and total-RNA-seq experiments. I.A.S. and Q.W. performed and analysed qRT-PCR. I.A.S. designed and conducted high-content microscopy. I.A.S. and G.P. performed the gene-pairing analysis. M.-H.S., S.B. and D.S. performed the bioinformatic analyses. D.S. performed the gene expression and splicing analysis. M.-H.S., I.A.S., Q.W. 
and M.D. analysed the genomic data. I.A.S. prepared genomic browser shots and schematics. I.A.S., M.-H.S. and M.D. prepared the figures. I.A.S., M.-H.S., D.S., G.P., G.L.H. and M.D. wrote the manuscript.

\section{Additional information}

Accession Codes The data discussed in this publication have been deposited in NCBI's Gene Expression Omnibus under the accession number GSE77917.

Supplementary Information accompanies this paper at http://www.nature.com/ naturecommunications

Competing financial interests: The authors declare no competing financial interests.
Reprints and permission information is available online at http://npg.nature.com/ reprintsandpermissions/

How to cite this article: Wang, Q. et al. Cajal bodies are linked to genome conformation. Nat. Commun. 7:10966 doi: 10.1038/ncomms10966 (2016).

(c) (i) This work is licensed under a Creative Commons Attribution 4.0 cc International License. The images or other third party material in this article are included in the article's Creative Commons license, unless indicated otherwise in the credit line; if the material is not included under the Creative Commons license, users will need to obtain permission from the license holder to reproduce the material. To view a copy of this license, visit http://creativecommons.org/licenses/by/4.0/ 